\title{
de Haas-van Alphen oscillations and magnetic breakdown: Semiclassical calculation of multiband orbits
}

\author{
Jean-Yves Fortin \\ Laboratoire de Physique Quantique, CNRS-URA 5626, Université Paul Sabatier, Toulouse, France \\ and Laboratoire de Physique Théorique, CNRS-URA 1436, Ecole Normale Superieure de Lyon, 46 Allée d'Italie, 69364 Lyon Cédex 07, \\ France \\ Jean Bellissard \\ Laboratoire de Physique Quantique, CNRS-URA 5626, Université Paul Sabatier, Toulouse, France \\ Miguel Gusmão \\ Laboratoire de Physique Quantique, CNRS-URA 5626, Université Paul Sabatier, Toulouse, France \\ and Universidade Federal do Rio Grande do Sul, Porto Alegre, Brazil \\ Timothy Ziman \\ Laboratoire de Physique Quantique, CNRS-URA 5626, Université Paul Sabatier, Toulouse, France \\ and Institut Laue Langevin, Boîte Postale 156, 38042 Grenoble Cédex, France
}

(Received 23 April 1997)

\begin{abstract}
We use an algebraic method to compute de Haas-van Alphen oscillations in two-dimensional systems in the semiclassical approximation for cases where the Fermi surface lies on more than one sheet of the energy surface. We treat magnetic breakdown by computing the Riemann surface associated with the Bloch energy equation. The topology of this surface, in particular, its fundamental group, is used to classify electronic trajectories in the complexified Brillouin zone. Three examples taken from tight-binding models of quasi-twodimensional organic conductors show how this can be implemented to calculate frequencies and breakdown fields. [S0163-1829(98)04803-6]
\end{abstract}

\section{INTRODUCTION}

The most precise way of measuring the Fermi surface in a metal is by measuring the behavior of thermodynamic and transport properties in magnetic fields varying in strength and direction. ${ }^{1}$ For the most part, the strength of fields available is sufficiently weak on an electronic scale that a semiclassical description is entirely satisfactory to explain the results. This semiclassical approach leads to the prediction of oscillations in the magnetization or resistance that are periodic in inverse field, with periodicity that is simply expressed, in appropriate units, as the area of extremal closed orbits on the Fermi surface perpendicular to the direction of the applied field. In addition, the amplitude of such oscillations in the case of magnetization is simply related to the effective mass of the carriers. It was realized in the early 1960's, in studies of metals such as Magnesium and Zinc, that to explain all the oscillations that one sees it is necessary to supplement the simplest semiclassical description of electrons confined to the Fermi surface with magnetic breakdown, ${ }^{2,3}$ that is, magnetic-field-induced tunneling from one part of the Fermi surface to another, in order to give oscillations corresponding to larger orbits than expected from the band structure. A semiempirical approach developed by Pippard ${ }^{4,5}$ and others culminated in a classification of orbits with tunneling probabilities from portion to portion of the Fermi surface depending on the local geometry. This was successful in explaining the different frequencies observed for a variety of metals. The question of interference between different orbits is rather more subtle. Stark and Friedberg ${ }^{6}$ predicted interference effects between different orbits in the transport but not the orbital magnetization.

More recently there has been a revival in what is called " "Fermiology:", that is to say the study of the geometry of the Fermi surface with magnetic means. This has been driven primarily by the study of high-quality samples of increasingly complex electronic structure, with larger unit cells, and by the availability of larger magnetic fields. There is also an impetus from the study of two-dimensional and quasi-twodimensional samples, where things should be simpler. On the theoretical side, especially in two dimensions, there has been progress with numerical means that allow one to test semiclassical models more quantitatively. There is room for improvement even in our understanding of the problem of noninteracting electrons. We want to extract reliable information about, for example, open orbits on the Fermi surface. Such orbits are not visible in the undamped oscillations, but they appear in breakdown phenomena. These orbits may be central to understanding the electronic instabilities towards charge- and spin-density waves. For example, in the quasitwo-dimensional organics, fine details in the shape of the open orbits ${ }^{7}$ are determinant to the predicted weak-coupling instabilities. It is important to disentangle essentially singleparticle effects of magnetic breakdown from collective effects such as field-induced changes in the magnetic structure.

In this paper we reanalyze within a semiclassical approach the problem of magnetic tunneling in two dimensions. We show that it is possible to calculate in a more 
systematic way than has been used previously (for example, in applying Chambers' formula ${ }^{5}$ ) the tunneling properties of an electron when the Fermi surfaces are described by roots of finite-degree polynomials, as is the case for any simple description in terms of tight-binding (or, equivalently, extended Hückel) models. In particular, experiments on the organic conductors are normally interpreted in terms of such models. This approach has several advantages: instead of having several semiempirical formulas to choose from, there is a unique answer. Commonly, Chambers' formula can be applied in slightly different forms, depending on the local curvature of orbits on the Fermi surface or, alternatively, the energy gap in the classically forbidden region of $\mathbf{k}$ space. One can also avoid questions that turn out to be irrelevant, such as "by what path does the electron tunnel," and what is the corresponding area, as the answer depends on an action that is independent of path in a given topological class, and the "area" in the case of real closed orbit becomes the real part of this action in the general case. As well as finding precise predictions for the breakdown field, this approach allows us to go beyond the simple junction models for magnetic breakdown. We shall discuss possible explanations of "forbidden" frequencies in the de Haas-van Alphen oscillations: those that apparently contradict the simplest classical intuition but that have been seen in numerical studies and deduced from experiment.

In the next section, Sec. II, we will review calculations of the single-band breakdown. In Sec. III we introduce the "algebraic formulation" of the multiband case. The final sections deal with several examples applying this formulation.

\section{TUNNELING FOR A SINGLE-BAND MODEL AND CHAMBERS' FORMULA}

The one-dimensional problem of tunneling between two regions separated by a gap was studied a long time ago. Kramers ${ }^{8}$ gave the semiclassical connection formulas for the problem of a particle of energy $E$ in a potential $V(x)$. These formulas connect wave functions in classically allowed and forbidden regions, where $V(x)$ is less than and greater than $E$, respectively. The transmission coefficient for the particle to go through a barrier in the region $a \leqslant x \leqslant b$ is, in the semiclassical limit,

$$
T \simeq \exp \{-2 \operatorname{Im}[S(a, b)] / \hbar\},
$$

where the action is $S(a, b)=\int_{a}^{b} p(x) d x, p^{2}(x)=2 m[E-$ $V(x)]$, and $a$ and $b$ denote the turning points at which the momentum $p$ vanishes. In one dimension, only one path joins $a$ to $b$, and so $T$ is unique. In two dimensions, the situation is different. A priori there are many paths which join $a$ to $b$ inside the gap. We might think then that a path integral theory would be necessary to compute the trajectory which minimizes the imaginary part of the action, and so maximizes $T$. In fact, as we will see in the next section, this is not necessary in the semiclassical approximation because $T$ depends on a topological invariant, and the contribution is, therefore, locally independent of the choice of the path. ${ }^{9}$

The simplest problem of magnetic breakdown in two dimensions is that of motion in a real hyperbolic potential with a saddle-point in the presence of an applied magnetic field. Fertig and Halperin ${ }^{10}$ exploited the analytical properties of the exact solutions of an harmonic wave function to compute the exact transmission coefficient of the problem. Our algebraic calculation of the multiband case is similar in spirit, in that it makes use of analytical continuation to complex wave vectors $\mathbf{k}$, but this is done in order to pass from sheet to sheet of the energy surfaces. Let us recapitulate their argument, in notation close to what we shall develop subsequently: we treat an electron in a two-dimensional periodic potential, and suppose that in the first Brillouin zone we have a saddle point, or, equivalently, a local expression for the oneelectron energies,

$$
\boldsymbol{\epsilon}(\mathbf{k})=\frac{\hbar^{2}}{2 m}\left(k_{x}^{2}-\lambda^{2} k_{y}^{2}\right)
$$

The solutions of $\epsilon(\mathbf{k})=C$, a positive constant, consist of two disconnected trajectories separated by a gap across which the particle could tunnel along the $k_{x}$ direction. Setting $l_{m}^{-2}$ $=e B / \hbar c$ as the magnetic length, and the operator $\hat{\mathbf{K}}=\boldsymbol{\nabla} / i$ $+e \mathbf{A} / \hbar c$, with the Landau gauge $\mathbf{A}=B(-y, 0,0)$, the quantization of the Hamiltonian leads to

$$
\hat{H}=\frac{\hbar^{2}}{2 m}\left\{\left(i \partial_{x}+l_{m}^{-2} y\right)^{2}-\lambda^{2}\left(i \partial_{y}\right)^{2}\right\}
$$

If we take $\hat{P}=\hat{K}_{x}$ and $\hat{X}=l_{m}^{2} \hat{K}_{y}$, with the commutation relations $[\hat{X}, \hat{P}]=i$, the identification with a one-dimensional problem of momentum $P$ and position $X$ is straightforward. In the space representation, $\hat{P}=-i \partial / \partial X$. By rescaling the $X$ variable as $X \rightarrow \sqrt{\lambda} X / l_{m}$, we may write the differential equation

$$
\left(\frac{\partial^{2}}{\partial X^{2}}+X^{2}+\frac{1}{4} k_{g}^{2} l_{m}^{2} / \lambda\right) \Psi(X)=0
$$

for the wave function $\Psi(X)$ of energy $E>0$, where $k_{g}$ $=2 \sqrt{2 m E} / \hbar$ is the gap in $\mathbf{k}$ space separating the two closest turning points at $k_{y}=0$. The case $E<0$ can be derived by using the symmetry $k_{x} \rightarrow \lambda k_{y}$ and $k_{y} \rightarrow k_{x} / \lambda$. Two linearly independent solutions of this differential equation are the odd and even parabolic cylinder functions

$$
\begin{gathered}
\Psi_{e}(X)=\exp \left(-\frac{i}{2} X^{2}\right) F\left(\frac{i}{16} k_{g}^{2} l_{m}^{2} / \lambda+\frac{1}{4}, \frac{1}{2} ; i X^{2}\right), \\
\Psi_{o}(X)=X \exp \left(-\frac{i}{2} X^{2}\right) F\left(\frac{i}{16} k_{g}^{2} l_{m}^{2} / \lambda+\frac{3}{4}, \frac{3}{2} ; i X^{2}\right),
\end{gathered}
$$

where $F$ is the confluent hypergeometric function. Applying $\hat{P}$ on $\exp \left( \pm i X^{2} / 2\right)$ one sees that this factor is associated with an incoming (-) or outgoing (+) current, respectively. To calculate the transmission coefficient, following Ref. 10, one forms the appropriate incoming, transmitted, and reflected wave packets (linear combinations of the two basis functions) near the saddle point. The correct relations between the coefficients of the wave function are computed by expanding the parabolic functions near $X=\infty$. The tunneling probability for the electron through the potential barrier $T$ $=\left|\left\langle\Psi_{\text {reflection }}, \Psi_{\text {incoming }}\right\rangle\right|^{2}$ is then 


$$
T=\frac{1}{1+\exp \left(\pi k_{g}^{2} l_{m}^{2} / 4 \lambda\right)} .
$$

Notice that we must use the reflected wave function and not the transmitted one to compute $T$ in the $(X, P)$-variable formulation, since the former represents physically the tunneling amplitude through the hyperbolic gap. Expressing $T$ in terms of the positive radius of curvature $R=k_{g} / 2 \lambda^{2}$ at the breaking points $\left( \pm k_{g} / 2,0\right)$, for weak field we find

$$
T \simeq \exp \left(-\frac{\pi}{2} \frac{l_{m}^{2} k_{g}^{3 / 2}}{\sqrt{2 / R}}\right)
$$

This is a special case of Chambers' expression $^{5}$ for the breakdown probability in the case of orbits symmetric around a saddle point. For the more general case Chambers replaced the factor $2 / R$ by $\left(1 / R_{+}-1 / R_{-}\right)$, the difference of the inverse radii at the nearest breaking points. For the case of equal radii (i.e., both convex or both concave with the same absolute radius of curvature), the Chambers' formula gives a $T$ that vanishes.

In the next section we shall show how to compute the tunneling probability in the case where we have several bands of a periodic potential.

\section{ALGEBRAIC FORMULATION}

\section{A. Semiclassical Hamiltonian operator in a magnetic field: Polynomial formulation}

Let us consider the Hamiltonian of a particle in a constant magnetic field $\mathbf{B}=B \mathbf{e}_{\mathbf{z}}$, perpendicular to a planar periodic potential $U(\mathbf{r})$,

$$
\hat{H}[\mathbf{A}]=-\frac{\hbar^{2}}{2 m}\left(\boldsymbol{\nabla}+\frac{i e}{\hbar c} \mathbf{A}\right)^{2}+U(\mathbf{r}) .
$$

We will work, as previously, with the Landau gauge. The potential is periodic: $U(\mathbf{r})=U\left(\mathbf{r}+\mathbf{R}_{m, n}\right)$, with $\mathbf{R}_{m, n} \equiv$ $m a_{x} \mathbf{e}_{\mathbf{x}}+n a_{y} \mathbf{e}_{\mathbf{y}}$. In the absence of a magnetic field, the wave functions are expressed in terms of Bloch functions $u_{s, \mathbf{k}}(\mathbf{r})$, with energy dispersion $\epsilon_{s}(\mathbf{k})$, where $s$ labels the bands in the first Brillouin zone. In the presence of a magnetic field, the Peierls approximation ${ }^{11}$ consists in taking the effective Hamiltonian $\hat{H}_{s}=\epsilon_{s}(\hat{\mathbf{K}})$, where $\hat{\mathbf{K}}=\boldsymbol{\nabla} / i+(e / \hbar c) \mathbf{A}(\mathbf{r})$. Here we shall restrict our discussion to Hamiltonians in which there is an underlying simple algebraic structure to the band dispersion. To show how this algebraic structure arises, consider the case of a linear superposition of single atomic orbitals. The periodic potential is written as a sum of local potentials, $U(\mathbf{r})=\sum_{m, n} v\left(\mathbf{r}-\mathbf{R}_{m, n}\right)$, with $v(\mathbf{r})$ decreasing rapidly away from the origin, and representing, for example, the potential or pseudopotential near a single atom. We can first compute the eigenfunctions $\phi_{m, n}(\mathbf{r}) \equiv \phi\left(\mathbf{r}-\mathbf{R}_{m, n}\right)$, solutions of the single-atom Schrödinger equation,

$$
\left\{-\frac{\hbar^{2}}{2 m} \nabla^{2}+v(\mathbf{r})\right\} \phi(\mathbf{r})=\epsilon_{0} \phi(\mathbf{r}),
$$

and then try to compute an approximate wave function of the periodic problem by forming a linear superposition $\Psi(\mathbf{r})$ $=\sum_{m, n} a_{m, n} \phi_{m, n}(\mathbf{r})$ for an energy $\epsilon .^{12}$ The result is expressed in the first Brillouin zone as a dispersion relation,

$$
\boldsymbol{\epsilon}(\mathbf{k})=\epsilon_{0}+\frac{\sum_{m, n} h_{m, n} \exp \left(i k_{x} m a_{x}+i k_{y} n a_{y}\right)}{\sum_{m, n} j_{m, n} \exp \left(i k_{x} m a_{x}+i k_{y} n a_{y}\right)},
$$

where

$$
\begin{gathered}
h_{m, n}=\int d^{2} r \phi_{0,0}^{*}(\mathbf{r})\left[v(\mathbf{r})-v\left(\mathbf{r}+\mathbf{R}_{m, n}\right)\right] \phi_{m, n}(\mathbf{r}), \\
j_{m, n}=\int d^{2} r \phi_{0,0}^{*}(\mathbf{r}) \phi_{m, n}(\mathbf{r}) .
\end{gathered}
$$

For a general periodic potential $U(\mathbf{r})$, a local potential can be defined as

$$
v(\mathbf{r}) \equiv U(\mathbf{r}) \frac{\sin \left(2 \pi x / a_{x}\right)}{2 \pi x / a_{x}} \frac{\sin \left(2 \pi y / a_{y}\right)}{2 \pi y / a_{y}} .
$$

For smooth $U$, the potential $v$ vanishes at large distances, and can be neglected for more than a few lattice spacings from the origin. The quantities $j_{m, n}$ and $h_{m, n}$ are rapidly decreasing. If we take all but a finite number of them to be zero, then $\epsilon$ is defined in terms of a complex polynomial of finite order in the variables $Z_{1} \equiv \exp \left(i k_{x} a_{x}\right)$ and $Z_{2} \equiv$ $\exp \left(i k_{y} a_{y}\right)$ :

$$
P\left(Z_{1}, Z_{2}, \epsilon\right)=0 \text {. }
$$

The condition that the polynomial $P$ of the complex variables $Z_{1}$ and $Z_{2}$ be constant defines a complex curve. In more general cases, for example, wave functions that are a linear combination of several orbitals for each atom, or when there are several atoms per unit cell, the same formulation is applicable: the polynomial simply becomes of higher order. Thus the restriction that we make to have different parts of the Fermi surface defined by roots of a single finite-order polynomial is rather mild.

This formulation is useful when we apply the Peierls substitution. Indeed, since the components of the operator $\hat{\mathbf{K}}$ are noncommutative,

$$
\left[\hat{K}_{x}, \hat{K}_{y}\right]=-i \hbar_{\mathrm{eff}} \equiv-i \frac{e B}{\hbar c},
$$

the substitution of operators in a general energy dispersion is not unique. If we expand the dispersion $\epsilon_{s}(\mathbf{k})$ in a Fourier series, we can define a choice

$$
\hat{H}_{s}=\sum_{\mathbf{a}=\mathbf{R}_{m, n}} \boldsymbol{\epsilon}_{s, \mathbf{a}} \exp (i \mathbf{a} \cdot \hat{\mathbf{K}}) .
$$

Generally, and for the examples we will study, the coefficients of the polynomial $P$ depend on the energy. The simplest example is when only the constant term depends linearly on energy.

\section{B. Riemann surfaces and the topological classification of closed electronic trajectories}

Consider now a closed-space trajectory at constant energy in a single band under the action of a magnetic field. The Bohr-Sommerfeld rule quantifies the action $\hbar \oint \mathbf{k} . d \mathbf{r}$ along 
this trajectory, or equivalently, the ratio between the total flux through the closed path and the quantum flux $\phi_{0}$ $=h c / e$. When the magnetic field is along a symmetry direction $z, k_{z}$ is constant. The Bohr condition then becomes

$$
\frac{\hbar c}{e B} \operatorname{Re} \oint_{\epsilon_{s}=\epsilon} K_{y} d K_{x}=2 \pi(n+\gamma), \quad n=0,1,2, \ldots,
$$

the contour of integration being taken in the clockwise direction. The constant $\gamma$ in the quantization condition is called the Maslov index. It can be computed to be $1 / 2$ by an expansion of $\boldsymbol{\epsilon}_{s}$ for small $\mathbf{k}$ near an assumed quadratic minimum (or for a hole near its maximum), where we can solve exactly a free-electron Hamiltonian. Quantization of the action is equivalent to quantization of energy. In the de Haas-van Alphen effect, the oscillations of the magnetization at low temperatures are determined by the area of all closed electronic trajectories at the Fermi surface, the contributions of other electrons being mutually compensated. In three dimensions, only the extremal areas at the Fermi surface perpendicular to the magnetic field direction contribute to the oscillations. We can define a characteristic magnetic field $B_{c}$ $=\phi_{0} /\left(a_{x} a_{y}\right)$ to be the reference field for the validity of the semiclassical approximation, for which $B \ll B_{c}$. For a monoatomic solid with $a_{x}=a_{y}=1 \AA$, we would have $B_{c} \approx 4.16$ $\times 10^{5} \mathrm{~T}$, much larger than available fields. In molecular conductors the unit cell can be much larger, typically of order 10 $\AA$. Even then, for example, for the $\alpha$ phases of (BEDT-TTF) ${ }_{2} \mathrm{MHgXCN}_{4}$ (to be discussed in Sec. VI), $B_{c}$ is $4200 \mathrm{~T}$. With experimental fields less than $100 \mathrm{~T}$, breakdown should only be visible if the gaps happen to be unusually small, as is the case for the organic conductor ${ }^{13,14}$ $\kappa-(\mathrm{ET})_{2} \mathrm{Cu}(\mathrm{NCS})_{2}$. For the $\alpha$ phases of (BEDT-TTF) ${ }_{2} \mathrm{MHgXCN}$ the question of whether magnetic breakdown should be observable or not, is a key element in a debate about the existence of a reconstruction from a density wave at low temperatures. ${ }^{15}$ It is important to eliminate any uncertainty as to the predicted value of the breakdown field from the band structure. The principal frequencies $f_{s}$ appearing in oscillations of the magnetization $M=M(1 / B)$ are proportional to the area surrounded by closed paths $\gamma_{s}$ at the Fermi surface ${ }^{16}$ when no breakdown effects occur:

$$
f_{s}=B_{c} \frac{a_{x} a_{y}}{(2 \pi)^{2}} \oint_{\gamma_{s}} K_{y} d K_{x} .
$$

For the case of orbits coupled by breakdown we should apply the Falicov formula, ${ }^{17}$

$$
\begin{aligned}
R_{s} & =\prod_{j=1}^{n_{s, t}} i p_{s, j} \prod_{j=1}^{n_{s, r}} q_{s, j}, \\
\prod_{j=1}^{n_{s, t}} p_{s, j} & =\exp \left[-2 \pi \operatorname{Im}\left(f_{s}\right) / B\right],
\end{aligned}
$$

introducing the amplitude factor $R_{s}$ for each closed electron orbit, which is assumed to pass by a number of breakdown points $n_{s, t}$ at which the electron tunnels, and a number $n_{s, r}$ where it does not. In Eq. (19), $p_{s, j}$ and $q_{s, j}$ are the absolute amplitudes of transmission and reflection for the $s$ path at each breakdown point, and all quantities are taken at the
Fermi energy, which is fixed. The imaginary part of the frequencies should obviously be positive at each point of breakdown along the trajectory. Moreover, the Maslov index depends on the energy susceptibility ${ }^{18}$ linearly with the quantity $B / B_{c}$. Thus, this term is in practice negligible. At fixed Fermi energy, we have a relation between the $K_{i}$, Eq. (17), which defines a constant-energy manifold. Provided we stay within this manifold, the integral in Eq. (18) is unchanged as the path is continuously deformed. ${ }^{9}$

Let us now consider possible breakdown effects between two different real $k$-space bands. To simplify notation, we will write the components of $\mathbf{K}$ in units of $(2 \pi) / a_{x}$ and $(2 \pi) / a_{y}$. The three-dimensional case along symmetry directions can be studied as in two dimensions, at fixed $K_{z}$. Let us finally remark that the total probability of transmission around a trajectory $s$ is, for small $B$,

$$
T_{s}=\exp \left\{-4 \pi \operatorname{Im}\left(f_{s}\right) / B\right\}=\prod_{i=1}^{n_{s, t}} \exp \left\{-B_{s, i} / B\right\}
$$

where the $B_{s, i}$ are the breakdown fields for each region of tunneling. Our aim here is to find a path that connects two bands via a tunneling effect. First, we construct the Riemann surface $M_{P}$ defined by $P$. We know from Ref. 19 that the solutions $Z_{2}=Z_{2}\left(Z_{1}\right)$ of $P=0$ form a multivalued algebraic function, so that to each point $Z_{1}$ of the complex plane are associated several possible values of $Z_{2}$. We can construct a surface on which this function is single valued. It is called the Riemann surface associated with $P$. The hyperelliptic functions are a special case where $P=Z_{2}^{2}-Q\left(Z_{1}, \epsilon\right), Q$ being a polynomial of finite degree in $Z_{1}$. If $n$ is the degree of $P$ in $Z_{2}$, then there are $n$ algebraic solutions, and so $M_{P}$ consists of $n$ sheets. To each point of the complex plane are associated $n$ equivalent points on the $n$ sheets [each identification $Z_{2}\left(Z_{1}\right)$ is associated to one sheet], defining the $n$ values of the function. Conversely, each point of the Riemann surface is associated with a unique value of $Z_{2}$. This provides the correct way to compute all continuous paths $\gamma(t)=\left\{Z_{1}(t), Z_{2}\left[Z_{1}(t)\right]\right\}, 0 \leqslant t \leqslant 1$. To construct $M_{P}$ we first compute the singularities of $P$. These are, for example, the points where we have a degeneracy in the otherwise distinct values of $Z_{2}\left(Z_{1}\right)$. There are two kinds of singular points: either $\{P=0, \nabla P=0$,$\} or \left\{P=0, \partial_{Z_{2}} P=0,\right\}$ the former case being more restrictive. The singularities indicate the presence of degeneracy among the different values of $Z_{2}$. Degeneracy is either because the multivalued complex curves $Z_{2}\left(Z_{1}\right)$ intersect at these points or else because an infinite slope occurs in the curve of $k_{x}$ as a function of $k_{y}$, for example. As we will see in some examples next, the latter case corresponds to the band edges. The procedure to remove the singularities and to set the branch cuts at which we paste the different sheets can be found, for example, in Refs. 19 and 20.

While points at infinity are not physically relevant here, they complicate the topological analysis. It is, therefore, convenient to compactify the space: we construct from $P$ a homogeneous polynomial as follows. Let $n_{P}$ be the total degree of $P$, i.e., the highest degree in $Z_{1}$ or $Z_{2}$, and let $Z_{1}$ $=Y_{1} / Y_{0}, Z_{2}=Y_{2} / Y_{0}$. Then $P$ can be expressed as 


$$
P\left(Z_{1}, Z_{2}, \epsilon\right)=\frac{1}{Y_{0}^{n_{P}}} Q\left(Y_{0}, Y_{1}, Y_{2}, \epsilon\right),
$$

where $Q$ is the appropriate homogeneous polynomial. If we consider the two-dimensional complex projective space $\mathrm{CP}^{2}$, formed by the equivalence classes $\left(Y_{0}, Y_{1}, Y_{2}\right)$ $\sim\left(\lambda Y_{0}, \lambda Y_{1}, \lambda Y_{2}\right)$, for $\lambda$ complex and non-zero, it is clear that $Q=0$ defines the same surface as $P=0$ in $\mathrm{CP}^{2}$. Points at infinity can be defined by setting $Y_{0}=0$. The Riemann sphere is defined as the manifold composed of the compactified complex plane. It is isomorphic to the sphere $S^{2}$ by stereographic projection.

We have the following connectivity property of $M(P)$ : if $P$ can be factorized in $n_{c}$ polynomials $P_{i}$, then $M(P)$ is the union of $n_{c}$ independent manifolds $M\left(P_{i}\right)$. The integer $n_{c}$ is called the connectivity index of the Riemann surface. Physically, an electron cannot tunnel between two paths $\gamma_{i}$ and $\gamma_{j}$ $(i \neq j)$ if they belong to two different Riemann surfaces. Let us suppose in the following that $M(P)$ is connected, and take any two different trajectories $\gamma_{i}$ and $\gamma_{j}$, with the constraints $\left\{\gamma_{i}(0)=\gamma_{j}(0)=P_{0}, \gamma_{i}(1)=\gamma_{j}(1)=P_{1}\right\}$. The action $S\left(\gamma_{i}\right)$ is equal to $S\left(\gamma_{j}\right)$ if and only if the two paths belong to the same homotopy class of the fundamental group $\pi_{1}\left(M(P), P_{0}, P_{1}\right) .{ }^{9}$ This is a consequence of Stoke's theorem: the difference between the integral taken along two trajectories that can be continuously deformed one to the other is an integral over the area defined by following one and returning on the other. On the submanifold of constant energy, this area is zero. As the de Haas-van Alphen frequencies are proportional to $S$, it is sufficient to choose one trajectory representative of each class of $\pi_{1}\left(M(P), P_{0}, P_{0}\right)$, which is in fact independent of the basis point $P_{0}$, and use it to compute the action $S$. It is clear that, in general, $S$ will be complex. The real part is identified with the real frequency. If there is no tunneling involved, it corresponds to the area $\int_{\gamma} \operatorname{Re}\left(K_{y}\right) d \operatorname{Re}\left(K_{x}\right)$ enclosed by the trajectory in the real $\left(K_{x}, K_{y}\right)$ plane. In that case $\left(Z_{1}, Z_{2}\right)$ belongs to the circle $S^{1} \times S^{1}$. If $S$ contains an imaginary part, this is the signature of a tunneling effect. In this case, the real part does not correspond exactly to the area in the first Brillouin zone. Indeed the contribution $-\int_{\gamma} \operatorname{Im}\left(K_{y}\right) d \operatorname{Im}\left(K_{x}\right)$ to the real part does not vanish in general. Moreover, the positive imaginary part of the action gives the total breakdown field.

Thus, we see that the question of the physical electronic trajectory in a process of tunneling is not relevant for the calculation of frequencies, and there is no weight associated to individual paths in a homotopy class: $S$ is a topological quantity invariant by a local change of path.

There is an important class of polynomials $P$ which can be written as $P\left(Z_{1}, Z_{2}\right)=Z_{1}^{n_{1}} Z_{2}^{n_{2}} P^{\prime}\left(Z_{1}+1 / Z_{1}, Z_{2}+1 / Z_{2}\right)$. Then, $P=0$ is equivalent to $P^{\prime}=0$, except at points $Z_{1}=0$ and $Z_{2}=0$. If, in addition, all coefficients of $P$ are real, we have symmetries in $S$ under transformations that simplify the analysis. For example, $Z_{1} \rightarrow 1 / Z_{1}$ implies $S \rightarrow-S$, and $Z_{1} \rightarrow 1 / \bar{Z}_{1}$ together with $Z_{2} \rightarrow 1 / Z_{2}$, imply $S \rightarrow-\bar{S}$. If we take a path $\gamma$ between two points $P_{0}$ and $P_{1}$, and the path $\gamma^{\prime}$ defined by

$$
\gamma^{\prime}(t)=\left[1 / \bar{Z}_{1}(t), 1 / \bar{Z}_{2}(t)\right]
$$

then the actions are mutually conjugate:

$$
S[\gamma]=\bar{S}\left[\gamma^{\prime}\right] .
$$

Therefore, we can restrict our calculations to the physical region on $M(P)$, i.e., $\operatorname{Im}(S) \geqslant 0$, corresponding to $\left|Z_{1}\right| \leqslant 1$.

A different but equivalent way to see these topological properties is to consider the homology/cohomology groups associated to the connected manifold $M(P)$. A closed electronic trajectory can be considered as a one-dimensional oriented cycle $c$ belonging to the set of such closed chains $C_{1}$. If $B_{1}$ is the set of boundaries, i.e., the one-dimensional chains $c$ that can be written as the boundary of twodimensional regions, we can define the simplicial homology of $M(P)$, the factor group $H_{1}=C_{1} / B_{1}$. It is clear that if $c_{i}$ and $c_{j}$ are two cycles belonging to the same class in $H_{1}$ they have the same action. On the other hand, we define the set $C^{1}$ of all one forms on $M(P)$ that are closed (the differentials are zero), and the set $B^{1}$ of all exact one forms (those that are differentials of functions). Then we can consider the de Rham cohomology group $H^{1}=C^{1} / B^{1}$. The inner product of a cycle $c$ of $C_{1}$ and $\omega=K_{y} d K_{x}$ of $C^{1}$,

$$
\pi(c, \omega)=\oint_{c} \omega,
$$

can be associated with the action $S$, and is, in fact, independent of the representative elements $c$ and $\omega$ in $H_{1}$ and $H^{1}$, so that in the semiclassical calculation $\pi$ acts on $H_{1} \times H^{1}$ as a scalar product, which remains constant for any small change of its argument $c$.

\section{APPLICATION TO A TWO-BAND MODEL}

\section{A. Model}

The model we consider in this section has a Fermi-surface shape close to the quasi-two-dimensional organic material that we will study in the next sections. By taking a polynomial of the lowest possible order, obtaining the singular points becomes completely elementary. In particular we will study the magnetic breakdown between an open Fermi surface and a closed circular area by computing the associated Riemann surface. Let us consider two planes coupled by a hybridization parameter $\alpha \geqslant 0$, in which the tight-binding constants in the $x$ and $y$ directions are $(-1,-1 / 2)$ for the first plane and $(-2,-2)$ for the second. We also introduce an energy gap $\epsilon_{g}$ between them. So, the Bloch Hamiltonian matrix can be written as

$$
\hat{H}=\left(\begin{array}{cc}
\epsilon_{1}\left(K_{x}, K_{y}\right) & \alpha \\
\alpha & \epsilon_{2}\left(K_{x}, K_{y}\right)
\end{array}\right),
$$

where

$$
\begin{gathered}
\epsilon_{1}\left(K_{x}, K_{y}\right)=-\cos \left(K_{x}\right)-1 / 2 \cos \left(K_{y}\right), \\
\epsilon_{2}\left(K_{x}, K_{y}\right)=-\epsilon_{g}-2\left[\cos \left(K_{x}\right)+\cos \left(K_{y}\right)\right] .
\end{gathered}
$$

Here we write $K_{x}$ and $K_{y}$ in units where the elementary lattice vectors are of unit length. Results will appear as fractions of the area of the first Brillouin zone. The Bloch bands at the Fermi energy are easily found by computing the secular equation, 


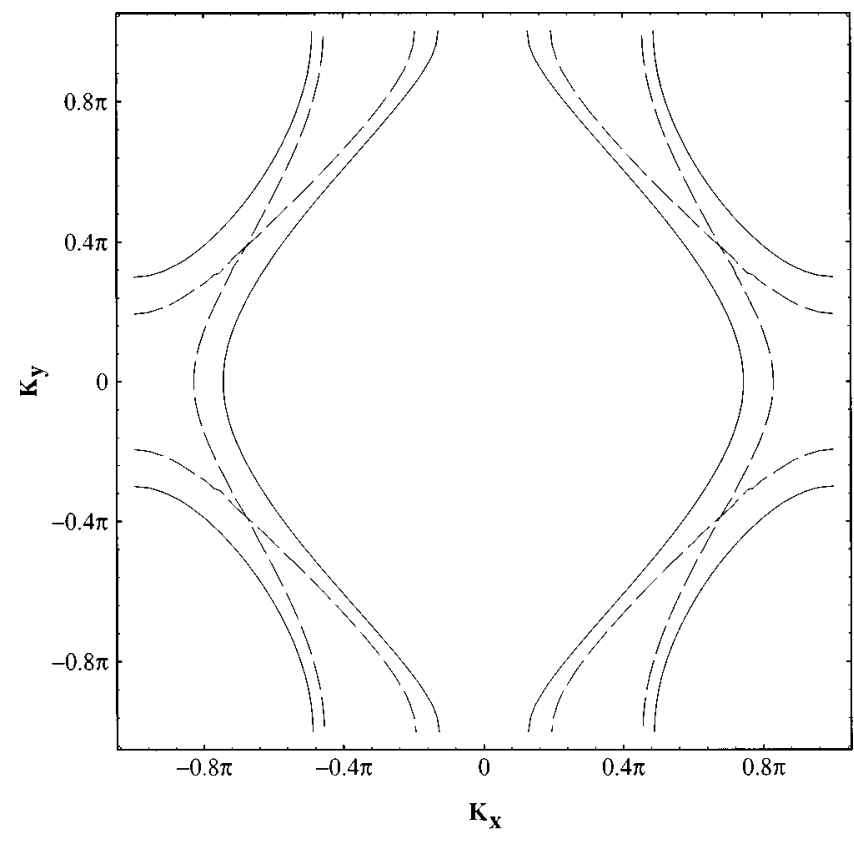

FIG. 1. The Fermi surface for energy $\epsilon_{F}=0.35$ for the two-band model of Sec. IV, with gap energy $\epsilon_{g}=0$ and hybridization $\alpha$ $=0.4$ (full lines), and the unhybridized case $\alpha=0$ (dashed lines).

$$
\operatorname{det}\left(\hat{H}-\epsilon_{F} \hat{I}\right)=0 .
$$

Let us first consider the case $\epsilon_{g}=0$ and $\alpha=0.4$. We will work with a filling ratio of 0.6 , which fixes the Fermi energy $\epsilon_{F}$ for zero field at approximatively 0.35 . The corresponding Fermi surface (see Fig. 1) has two open orbits along the $K_{y}$ direction and four rounded pieces at the corners. The dashed lines in Fig. 1 correspond to $\alpha=0$ at the same energy. In this case, there are degeneracies at four points in the Brillouin zone, and a nonzero $\alpha$ lifts these degeneracies. The determinant can be transformed into the polynomial

$$
\begin{aligned}
P\left(Z_{1}, Z_{2} ; \alpha, \epsilon_{F}\right)= & 2\left[\left(Z_{1}^{2}+1\right) Z_{2}+\frac{1}{2}\left(Z_{2}^{2}+1\right) Z_{1}+2 \epsilon_{F} Z_{1} Z_{2}\right] \\
& \times\left[\left(Z_{1}^{2}+1\right) Z_{2}+\left(Z_{2}^{2}+1\right) Z_{1}+\epsilon_{F} Z_{1} Z_{2}\right] \\
& -4 \alpha^{2} Z_{1}^{2} Z_{2}^{2} .
\end{aligned}
$$

The degree of this polynomial is 4 , which corresponds to the number of identifications of the algebraic function $Z_{2}$ $=Z_{2}\left(Z_{1}\right)$. Indeed, there are $K_{x}$ 's in Fig. 1 for which there are four distinct solutions of $K_{y}$. Therefore, in order to define a surface on which $Z_{2}$, considered as a function of $Z_{1}$, is single valued, we must take at least four copies of the complex plane. The different identifications will be noted $Z_{2}[ \pm 1, \pm 1]\left(Z_{1}\right)$. As we have discussed, and in order to be able to draw the Riemann surface, we use compactified planes that are represented by surfaces of spheres. In Fig. 2 we draw four complex planes labeled in this way. In each case the point at infinity is behind the visible part of the sphere. We draw the unit circles corresponding to possible real values of $K_{y}$ on each sphere. Let us now compute the singular points defined by

$$
P\left(Z_{1}, Z_{2} ; \alpha, \epsilon_{F}\right)=0, \quad \partial_{Z_{2}} P\left(Z_{1}, Z_{2} ; \alpha, \epsilon_{F}\right)=0 .
$$

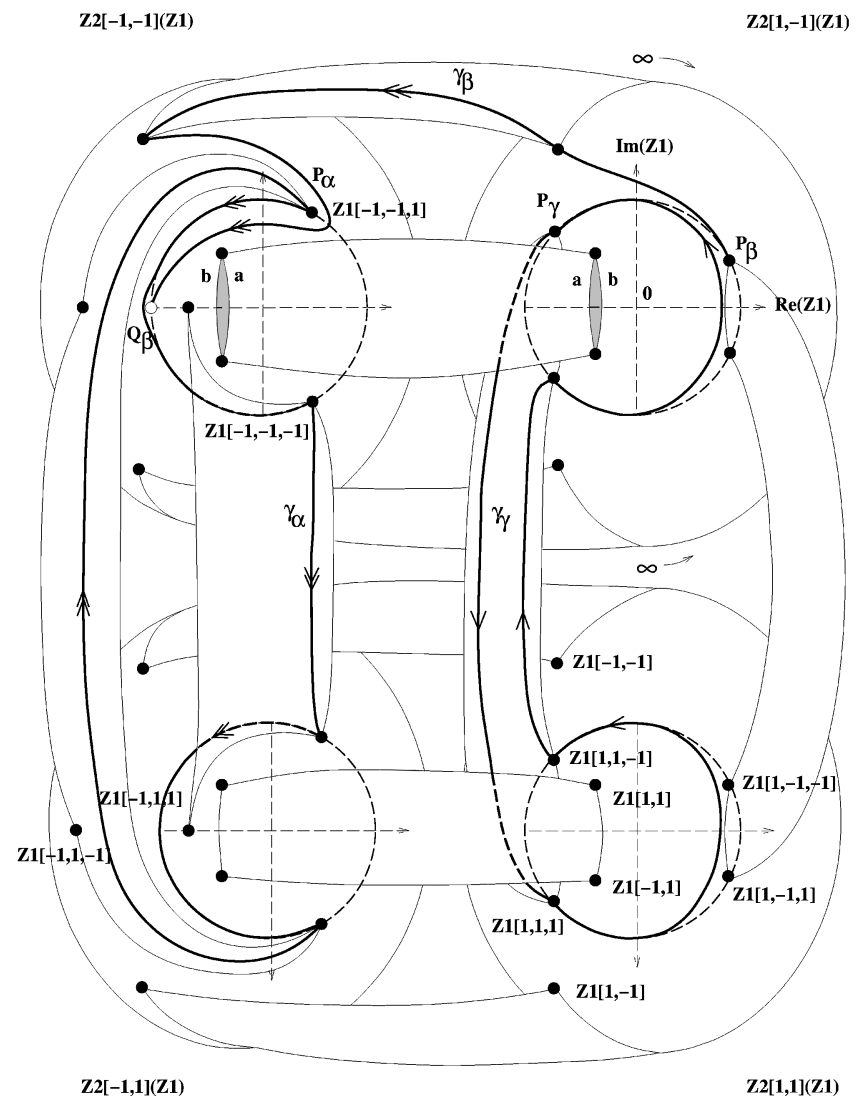

FIG. 2. Riemann surface for the two-band model with the parameters of Fig. $1, \epsilon_{F}=0.35, \epsilon_{g}=0$, and $\alpha \simeq 0.4$. The singular points are indicated by large dots, and the unit circles by lines on each of the four Riemann spheres. The spheres are connected by handles as described in the text. For simplicity, we show the gluing together of only two lines of branch cuts between pairs of singularities in the upper Riemann spheres. The different orbits $\alpha, \beta$, and $\gamma$ are indicated, and are drawn projected onto real space in Fig. 3.

This polynomial has twelve singular points, and near each of these $\left(Z_{1}=Z_{a}\right)$, the singular part of $Z_{2}$ behaves as $\sqrt{Z_{1}-Z_{a}}$. This defines the ramification index as being 2 . At these points, two sheets are connected. If $X_{i} \equiv Z_{i}+1 / Z_{i}, i=1,2$, then

$$
\begin{gathered}
Z_{2}\left[\sigma, \sigma^{\prime}\right]=\frac{1}{4}\left[-\left(3 X_{1}+5 \epsilon_{F}+\epsilon_{g}\right)+\sigma \sqrt{q\left(X_{1}\right)}\right. \\
\left.+\sigma^{\prime} \sqrt{q_{\sigma}\left(X_{1}\right)}\right], \\
q\left(X_{1}\right)=\left(X_{1}+3 \epsilon_{F}-\epsilon_{g}\right)^{2}+16 \alpha^{2}, \\
q_{\sigma}\left(X_{1}\right)=\left(3 X_{1}+5 \epsilon_{F}+\epsilon_{g}-\sigma \sqrt{q\left(X_{1}\right)}\right)^{2}-16 .
\end{gathered}
$$

The singular points occur where the the arguments of the square roots vanish. Indeed, if $q=0$, then $Z_{2}\left[1, \sigma^{\prime}\right]=Z_{2}$ $\left[-1, \sigma^{\prime}\right]$, for $\sigma^{\prime}= \pm 1$, and if $q_{\sigma}=0, Z_{2}[\sigma, 1]=Z_{2}[\sigma,-1]$. The condition $q=0$ provides us with four solutions, $Z_{1}[\eta$, $\pm 1]$, which are solutions of $X_{1}=-3 \epsilon_{F}+\epsilon_{g}+i 4 \eta \alpha$, with $Z_{1}[\eta,+1]$ denoting the solution for which the real part is greater than that of $Z_{1}[\eta,-1]$. Vanishing $q_{\sigma}$ leads to four solutions for each $\sigma, Z_{1}[\sigma, \eta, \pm 1]$, which satisfy the equality $X_{1}=\frac{1}{2}\left[-3 \epsilon_{F}-\epsilon_{g}-3 \eta+\sigma \sqrt{\left(-\epsilon_{F}+\epsilon_{g}+\eta\right)^{2}+8 \alpha^{2}}\right]$, with the solutions $Z_{1}[\sigma, \eta, 1]$ and $Z_{1}[\sigma, \eta,-1]$ ordered in 


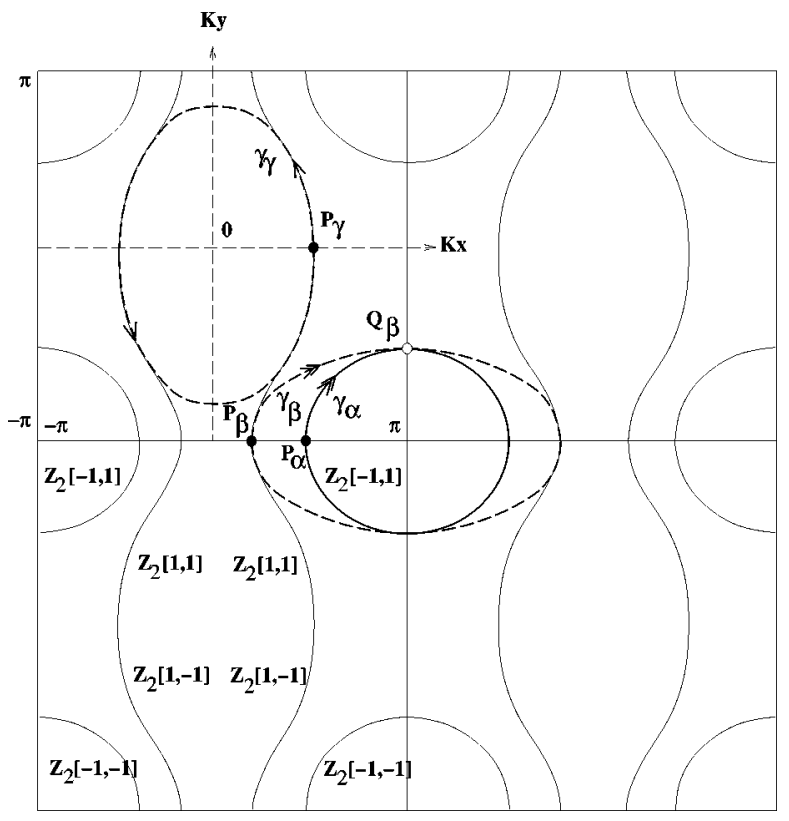

FIG. 3. The extended Brillouin zone in real $k$ space for the two-band model for the same parameters as Figs. 1 and $2(\alpha$ $=0.4, \epsilon_{g}=0$, and $\epsilon_{F}=0.35$ ), showing the projection of the orbits $\gamma_{\alpha}, \gamma_{\beta}$, and $\gamma_{\gamma}$, and the identifications $Z_{2}$.

decreasing order of real parts (or, in the case of equality, of the imaginary parts). The distribution of the singular points is indicated on the Riemann surface in Fig. 2. The different sheets are represented by a Riemann sphere on which we have drawn the unit circle and, with black circles, marked the singular points around it. In particular, we recognize on the unit circle the points delimiting the real bands $K_{y}=K_{y}\left(K_{x}\right)$ by a vertical tangent (Fig. 1). $Z_{1}=0$ is a singular polar point that does not play any role in the Riemann surface construction. It is clear that for $Z_{1}=0$ or $Z_{1}=\infty$ the imaginary part of the action is infinite, so that the wave function should vanish. Since the Hamiltonian has many symmetries $\left(Z_{1} \rightarrow 1 / Z_{1}\right.$, $Z_{1} \rightarrow \bar{Z}_{1}$ ), we only need to count the points inside the unit disk on the upper half complex plane to deduce the others. In fact, only five points of the twelve need to be computed. Only one branch cut comes out at each singular point, since the ramification index is 2 for each of them. Thus we join pairs of singular points by a segment in order to close the branch cuts. Then, we glue two sheets along a corresponding segment by cutting it in two parts, $\mathbf{a}$ and $\mathbf{b}$, and identifying the suitable edges (delimiting the dark area on Fig. 2) by the process indicated, for example, in Ref. 19: if we take a path beginning at a point $P$ in the neighborhood of a singular point, and if we turn twice around this point (the ramification index being 2), we have to go back to the original point $P$. We obtain, finally, a surface of genus $g=5$, which allows us to compute all the homotopy classes.

\section{B. The fundamental frequencies}

In this section we compute three fundamental frequencies that appear in the Fourier spectrum of the magnetization for the parameters $\alpha=0.4, \epsilon_{F}=0.35$, and $\epsilon_{g}=0$. We have drawn a representative path of each of them $\left(\gamma_{\alpha}, \gamma_{\beta}, \gamma_{\gamma}\right)$ on the Riemann surface (Fig. 2) and in the real $\mathbf{k}$ space (Fig. 3).
TABLE I. Values of the fundamental de Haas-van Alphen frequencies for each value of the hybridization parameter $\alpha$, and the corresponding Fermi energy $\epsilon_{F}$, for a fixed filling of 0.6.

\begin{tabular}{lcccc}
\hline \hline$\epsilon_{F}$ & $\alpha$ & $f_{\alpha} / B_{c}$ & $f_{\beta} / B_{c}$ & $f_{\gamma} / B_{c}$ \\
\hline 0.341 & 0.3 & 0.286 & $0.412+2.90 \times 10^{-2} i$ & $0.486+1.77 \times 10^{-2} i$ \\
0.349 & 0.4 & 0.264 & $0.411+4.71 \times 10^{-2} i$ & $0.465+1.18 \times 10^{-2} i$ \\
0.363 & 0.5 & 0.242 & $0.410+6.75 \times 10^{-2} i$ & $0.442+5.17 \times 10^{-3} i$ \\
\hline \hline
\end{tabular}

The comparison between the two figures helps us to understand how the complex electron- or hole-trajectory scheme works. The double arrows designate a hole trajectory, and the single arrows, an electronic one. This means that the $\alpha$ orbit surrounds a maximum peak in energy, with a negative effective mass. In Fig. 2 the $\alpha$ orbit can be followed by starting at the point $P_{\alpha}$ on the upper left part of the sphere $Z_{2}[-1,-1]$ and moving towards $Q_{\beta}$ (the path is shown slightly to the left near this point but this is just to avoid confusion with the trajectory $\gamma_{\beta}$ ). It continues on the unit circle until the singular point $Z_{1}[-1,-1,-1]$ where it must move onto the other sphere in the lower left of the figure. Similarly it moves around part of the unit circle and returns to $P_{\alpha}$. The handles are drawn to show the topology but note that there is no contribution to the action from the passage from one surface to the other. As the whole path is on the unit circles the $k$-vectors are real throughout. As this trajectory does not include any magnetic breakdown, its frequency $f_{\alpha}$ dominates at low field. As the field increases, magnetic breakdown appears between hole pockets and the open electronic orbits $\left(\gamma_{\beta}\right)$, and between the open orbits themselves $\left(\gamma_{\gamma}\right)$. The $\gamma_{\gamma}$ orbit is also shown in its entirety in Fig. 2, where it appears in the two right-hand spheres. Starting at the point $P_{\gamma}$ the path passes immediately to the lower right-hand sphere $Z_{2}[1,1]$ and describes part of the unit circle. At some point it leaves the circle, corresponding to the tunneling from the right hand part of the open surface to the left. Crossing the axis $K_{y}=0$ it moves onto the upper right-hand sphere $Z_{2}[1,1]$ and makes a second tunneling to return to $P_{\gamma}$. The imaginary part of the action comes from the two departures from the unit circles. For $\gamma_{\beta}$ we show only the first quadrant of the path from $P_{\beta}$ to $Q_{\beta}$. The path moves outside the unit circle in the upper right-hand sphere and passes via singular points to the upper left sphere and the point $Q_{\beta}$. In the intermediate range, a combination $\left(f_{\beta}+f_{\alpha}\right) / 2$ can appear because of the high degree of symmetry in the first Brillouin zone. The values of the different frequencies, computed for a filling rate of 0.6 , are listed in Table I.

Let us remark that for $\alpha=0.3$ we find $f_{\beta}=0.413-7.4$ $\times 10^{-4} i$, the imaginary contribution being negligible, and we observe that this is generally so. The breakdown fields for each tunneling region, i.e., the fields giving the probabilities of tunneling at each "junction" where the contours of different branches of the Fermi surface are close, are $B_{\beta, i}$ $=0.148(2)$ and $B_{\gamma, i}=0.074(2)$ (the integer in brackets indicates the number of breakdowns around one orbit), in units of $B_{c}$ and for $\alpha=0.4$. Varying the $\alpha$ values between 0.3 and 0.5 does not alter the topology of the Riemann surface. In fact, the singular points move on the Riemann sphere, but the connections between the sheets are not destroyed. The change of topology when $\alpha$ decreases to zero comes from the 


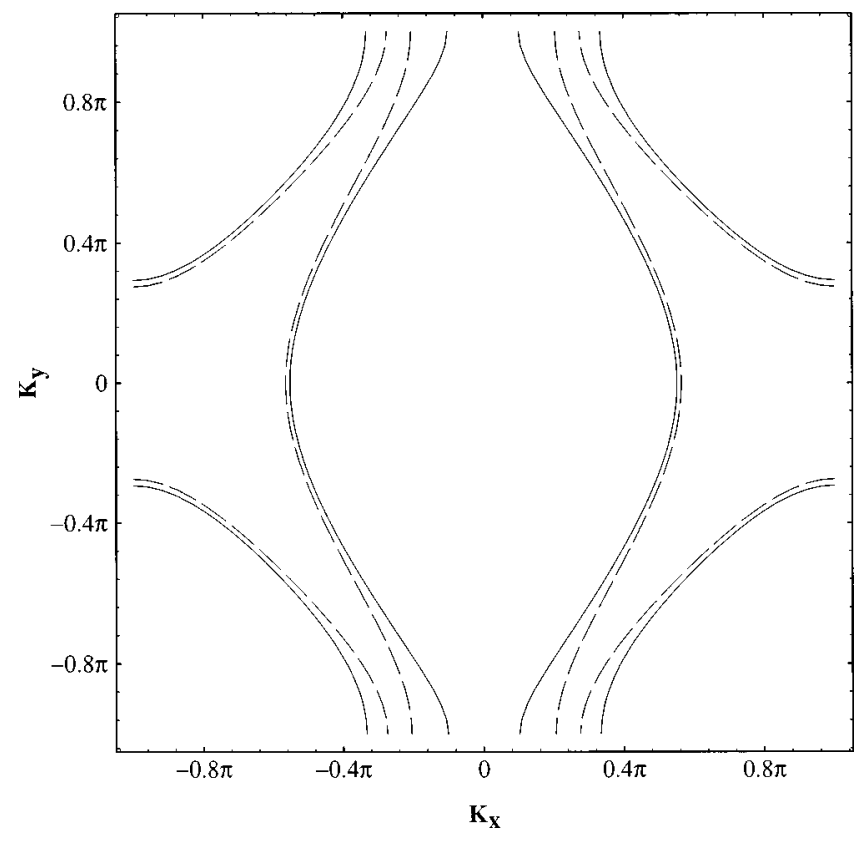

FIG. 4. Fermi surface for the two-band model for the case in which there is no crossing at zero hybridization. The parameters are $\alpha=0.3, \epsilon_{F}=-0.3$, and $\epsilon_{g}=1$ (full lines); $\alpha=0$, same $\epsilon_{F}$ and $\epsilon_{g}$ (dashed lines).

fact that two pairs of singular points $\left(Z_{a}, 1 / \bar{Z}_{a}\right)$ and $\left(\bar{Z}_{a}, 1 / Z_{a}\right)$ tend towards each other and merge to form a pair of conjugate points on the unit circle, $Z_{a}^{\lim }$ and $1 / Z_{a}^{\lim }$ $=Z_{a}^{\lim }$. When they merge, the singularities disappear because the ramification index is equal to 2. Indeed, when $\alpha$ decreases towards zero, $\left(Z_{1}[1,-1], Z_{1}[-1,1]\right)$ and $\left(Z_{1}\right.$ $\left.[-1,-1], Z_{1}[1,1]\right)$ collapse on the unit circle to $Z_{a}^{\lim }$ and $Z_{a}^{\text {lim }}$ at the intersections of the "real" bands in the Brillouin zone: $Z_{a}^{\lim }$ is at the intersections of the arcs joining $Z_{1}[1$, $-1,1]$ to $Z_{1}[1,1,1]$, and $Z_{1}[-1,-1,1]$ to $\exp (i \pi)$, respectively. Similarly, $Z_{a}^{\lim }$ is at the intersections of the arcs joining $Z_{1}[1,1,-1]$ to $Z_{1}[1,-1,-1]$, and $\exp (-i \pi)$ to $Z_{1}[-1$, $-1,-1]$. Therefore, in this limit, the Riemann surface breaks into two parts, $\left\{Z_{2}[-1,-1]\left(Z_{1}\right), Z_{2}[-1,1]\left(Z_{1}\right)\right\}$ and $\left\{Z_{2}[1,-1]\left(Z_{1}\right), Z_{2}[1,1]\left(Z_{1}\right)\right\}$. The path $\gamma_{\beta}$ can no longer be followed because $M(P)$ is disconnected. Moreover, the probability of tunneling for this path goes to 1 when $\alpha \rightarrow 0^{+}$, but seems to vanish at $\alpha$ exactly equal to zero. So, if we travel from $P_{\beta}$ to $Q_{\beta}$ (we consider one part of the path $\gamma_{\beta}$ ), the limit of $M(P)$ when $\alpha \rightarrow 0$ induces a rupture of the path. However, when we analyze Fig. 1, we see that the path should be naturally continuous (by inspection of the dashed lines). Moreover, the continuity of the path $P_{\alpha} \rightarrow Q_{\beta}$ following $\gamma_{\alpha}$ in the limit $\alpha \rightarrow 0^{+}$is not correct if we inspect the same figure. These observations tell us that the Riemann surface obtained as the limit $\alpha \rightarrow 0^{+}$of $M(P)$ is wrong, in the sense that it does not correspond to the Riemann surface we are expecting at $\alpha=0$. In fact, for $\alpha=0$, the original Hamiltonian is already diagonalized, so that we can construct two disconnected Riemann surfaces that do not correspond exactly to those obtained by the finite- $\alpha-$ ones in the limit $\alpha \rightarrow 0$. Indeed, the distribution of the singular points with respect to the disconnected parts of the Riemann surface is

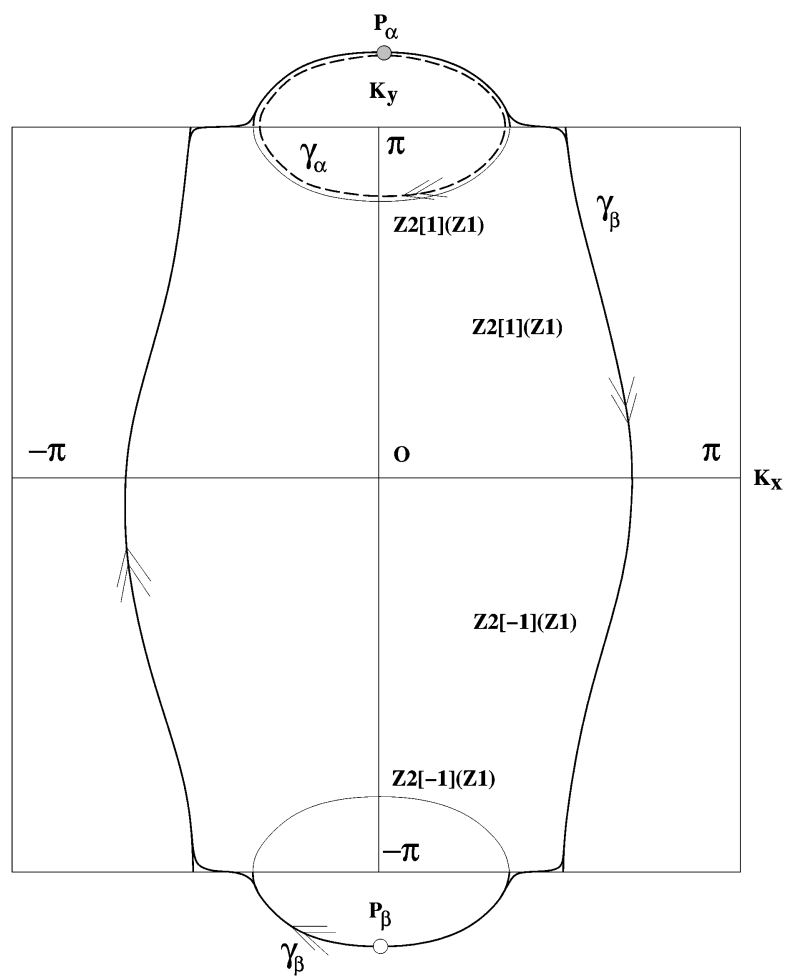

FIG. 5. Fermi surface of the simplified tight-binding model for the organic compound $\kappa$-(BEDT-TTF $)_{2} \mathrm{Cu}(\mathrm{NCS})_{2}$. The parameters are $c_{1}=100.75, c_{2}=70.75, b=60$, and $\epsilon_{F}=-63.52$ (in meV). As explained in the text, the difference between $c_{1}$ and $c_{2}$, and therefore the gap between surfaces at $K_{y}=\pi$, have been greatly exaggerated in order to compare to the numerical results of Figs. 6 and 7. The $\gamma_{\alpha}$ and $\gamma_{\beta}$ trajectories in real $\mathbf{k}$ space are indicated, as well as the identifications to be used in constructing the Riemann surface (Fig. 8).

different even if the overall set of values is the same. On these surfaces, we have the correct way of choosing the paths when we cross the degenerate point in Fig. 1, with a unit probability for the path $P_{\beta} \rightarrow Q_{\beta}$. On the other hand, the case $\epsilon_{g} \neq 0$ avoids the degeneracies that occur on the Fermi surface at $\alpha=0$, and now the limit of $M(P)$ when $\alpha \rightarrow 0^{+}$ corresponds to the correct result when $\alpha=0$. This is the next observation.

\section{The case $\epsilon_{g} \neq 0$ and the discontinuity of magnetic breakdown}

In Fig. 4 we have drawn the Fermi surface choosing the values $\alpha=0.3, \epsilon_{F}=-0.3, \epsilon_{g}=1$ (full lines), and the same with $\alpha=0$ (dashed lines). For this case no degeneracy point appears in the Brillouin zone in the limit $\alpha \rightarrow 0^{+}$. As previously, the Riemann-surface topology is identical for $\alpha \neq 0$, and the distribution of the 12 singular points is almost the same. The only difference is that when $\alpha$ decreases to zero the two previous pairs of singular points tend to two conjugate points on the unit circle, but instead of reaching the domain of the "real" band, they reach the "complex" region that does not appear in the Brillouin zone. That is why the calculation of the imaginary part of $\gamma_{\beta}$ leads to a nonvanishing value with a probability less than unity in the limit $\alpha \rightarrow 0$. At $\alpha=0$, this probability vanishes, as before, although 


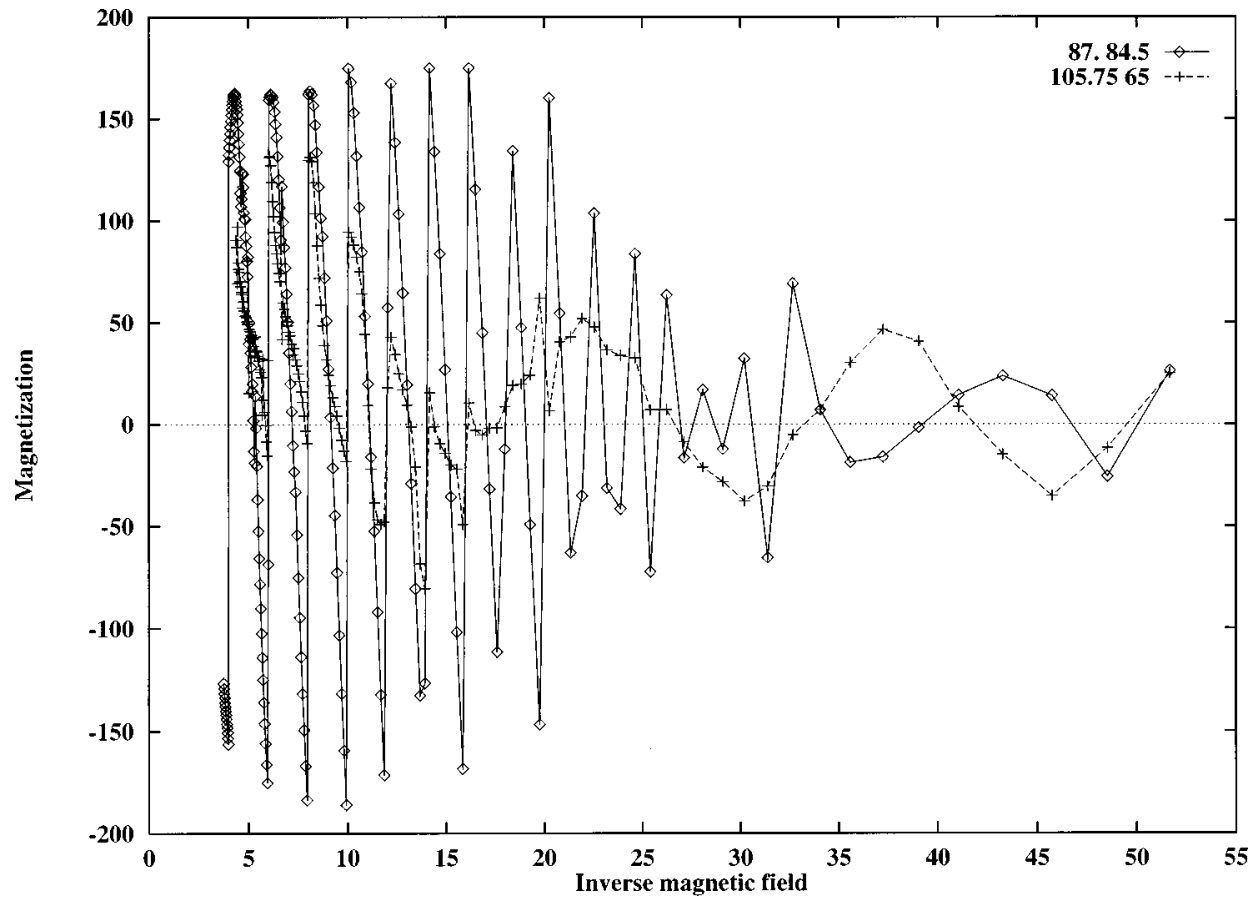

FIG. 6. Magnetization curves against $B_{c} / B$ for values of parameters $\left(c_{1}, c_{2}\right)$ corresponding to the fitted experimental values (87.5, 84.5), as well as the value $(100.75,70.75)$ corresponding to Figs. 5 and 7.

the similarity of the Fermi-surface shapes in the cases $\alpha$ $=0$ and $\alpha \neq 0$ could be misleading. Moreover, the Riemann surface is a continuous construction when $\alpha \rightarrow 0$. The fact that the singular points collapse, by symmetry of the Hamiltonian, at the unit circle and not at infinity (where the wavefunction amplitude should be zero) explains why the transmission coefficient between $P_{\beta}$ and $Q_{\beta}$ does not vanish in this limit. Therefore, we have shown a duality between two cases: (1) $\epsilon_{g}=0$-discontinuity between $\left.M(P)\right|_{\alpha \rightarrow 0^{+}}$and $\left.M(P)\right|_{\alpha=0}$; continuity of the transmission coefficient between $P_{\beta}$ and $Q_{\beta}$ along the path $\gamma_{\beta}$ (see Fig. 3); (2) $\epsilon_{g} \neq 0$ - continuity of $M(P)$ at $\alpha=0$, and discontinuity of the previous transmission coefficient.

What does this imply for magnetic oscillations, for the second case in particular? Strictly speaking we could say that Chambers' formula is inapplicable for $\alpha=0$, although in the

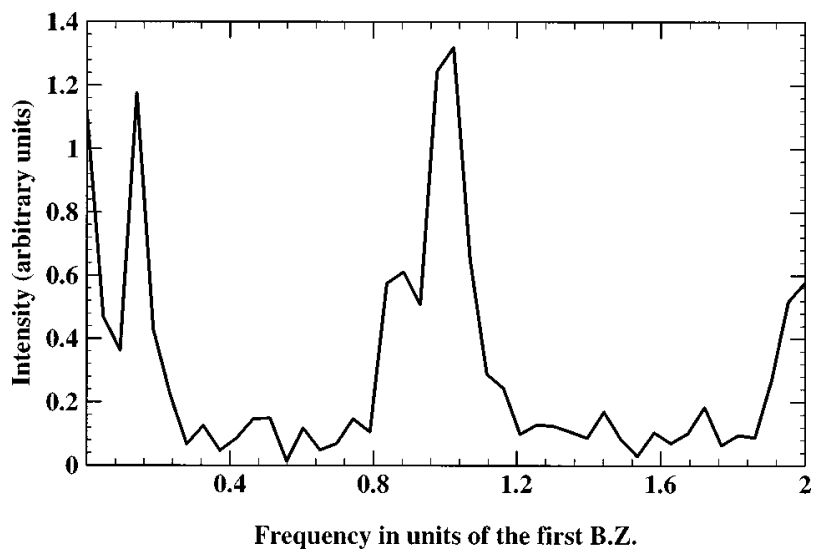

FIG. 7. Intensity of the Fourier components of the curve of Fig. 6 for the parameters $\left(c_{1}, c_{2}\right)=(100.75,70.75)$. The frequency $\beta$ $-\alpha$ is visible as a shoulder on the primary breakdown peak of frequency $100 \%$ of the first Brillouin zone. trivial sense that there is a selection rule that gives no breakdown. What is happening is that the critical field for nonzero $\alpha$ stays close to the value predicted by Chambers' formula, but the amplitude must vanish. We can visualize this physically for small $\alpha$ by imagining that a wave packet that approaches the point of breakdown will only tunnel if its velocity, set by the field $H$, is sufficiently small that tunneling can occur via the small coupling $\alpha$.

\section{APPLICATION TO THE TWO-DIMENSIONAL ORGANIC SUPERCONDUCTOR $\kappa$-(BEDT-TTF $)_{2} \mathrm{Cu}(\mathrm{NCS})_{2}$ : A TWO-BAND MODEL}

This quasi-two-dimensional organic superconductor has been experimentally studied by several groups. ${ }^{21}$ In particular, the influence of pressure on the different parameters of the magnetoresistance, such as the area of the small closed orbit and the effective mass, has been analyzed. Moreover, a correlation between the superconducting temperature and the effective mass has been measured. The band structure used to interpret the experimental results ${ }^{21}$ for this organic compound is based on an effective dimer model. Consider two dimers per unit cell, $A$ and $B$, whose overlap $A-B$ within the unit cell is $c_{1}$. In the $x$ direction, there is a coupling $b$ which is the direct overlap $A-A$ or $B-B$. A dimer $A$ at the origin has four neighboring dimers $B$, located diagonally around it. The overlap with the $B$ 's above is $c_{1}$, while that with those below is $c_{2}$. The experimental values for these parameters are (in meV) $b=60, c_{1}=87$, and $c_{2}=84.5$. The Fermi energy is $\epsilon_{F} \simeq-b$. For theoretical convenience, we will allow these values to change in order to deform the Fermi surface and vary the de Haas-van Alphen frequencies as pressure does. ${ }^{22}$ We can express the Bloch Hamiltonian as 


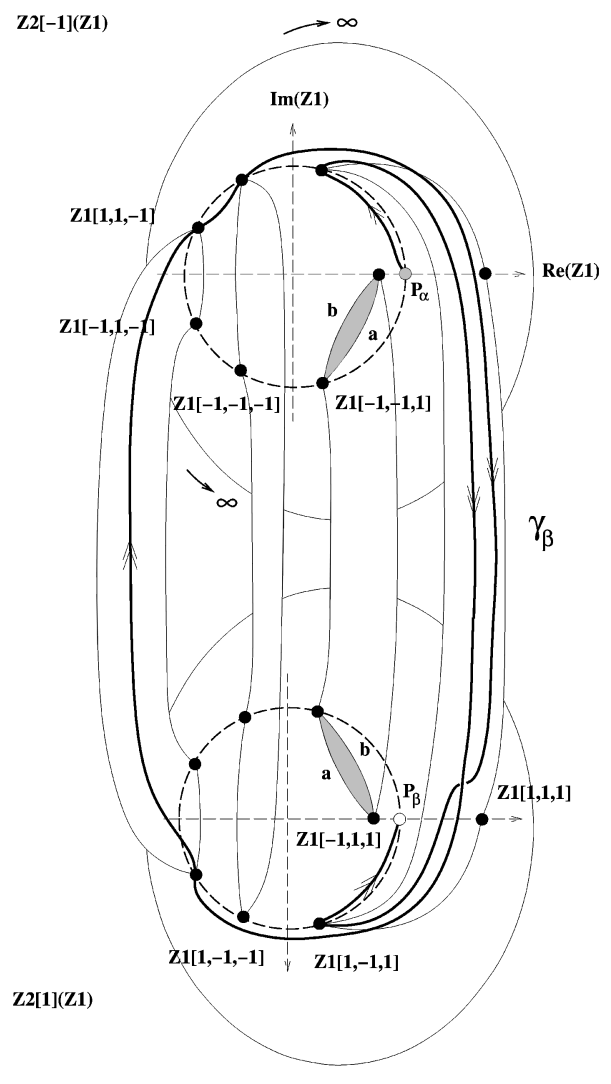

FIG. 8. Riemann surface for the tight-binding model of the organic conductor $\kappa$-(BEDT-TTF $)_{2} \mathrm{CU}(\mathrm{NCS})_{2}$. The two Riemann spheres are connected as indicated. The orbits of Fig. 5 are also shown.

$\hat{H}=\left(\begin{array}{cc}2 b \cos \left(K_{x}\right) & \left(1+e^{i K_{x}}\right)\left(c_{1}+c_{2} e^{i K_{y}}\right) \\ \left(1+e^{-i K_{x}}\right)\left(c_{1}+c_{2} e^{-i K_{y}}\right) & 2 b \cos \left(K_{x}\right)\end{array}\right)$,

yielding the dispersion relation

$$
\begin{aligned}
{\left[\epsilon-2 b \cos \left(K_{x}\right)\right]^{2}=} & 2\left[c_{1}^{2}+c_{2}^{2}+2 c_{1} c_{2} \cos \left(K_{y}\right)\right] \\
& \times\left[1+\cos \left(K_{x}\right)\right],
\end{aligned}
$$

which is symmetrical in $c_{1}$ and $c_{2}$. In the following, we will fix $b$ and $\left(c_{1}+c_{2}\right) / 2$, and vary the difference $\left(c_{1}-c_{2}\right)$. We also fix the filling ratio as one electron per site, i.e., we assume the stoichiometry to correspond to the experimental situation. We have drawn in Fig. 5 the Fermi surface for an arbitrary set of parameters (given in meV). The two surfaces are such that the sum of the closed hole-orbit area added to the inside area delimited by the open orbits is exactly one. Indeed, we first begin to fill the band that has the lowestenergy minimum, at $\epsilon \sim-300 \mathrm{meV}$, situated in the center of

TABLE II. Singular points of the Riemann surface corresponding to the dispersion relation (32).

\begin{tabular}{lcc}
\hline \hline \multicolumn{1}{c}{$\eta^{\prime}$} & 1 & -1 \\
\hline$Z_{1}\left[1,1, \eta^{\prime}\right]$ & 7.84 & $-0.956+0.295 i$ \\
$Z_{1}\left[1,-1, \eta^{\prime}\right]$ & $-0.216+0.976 i$ & $-0.717+0.697 i$ \\
\hline \hline
\end{tabular}

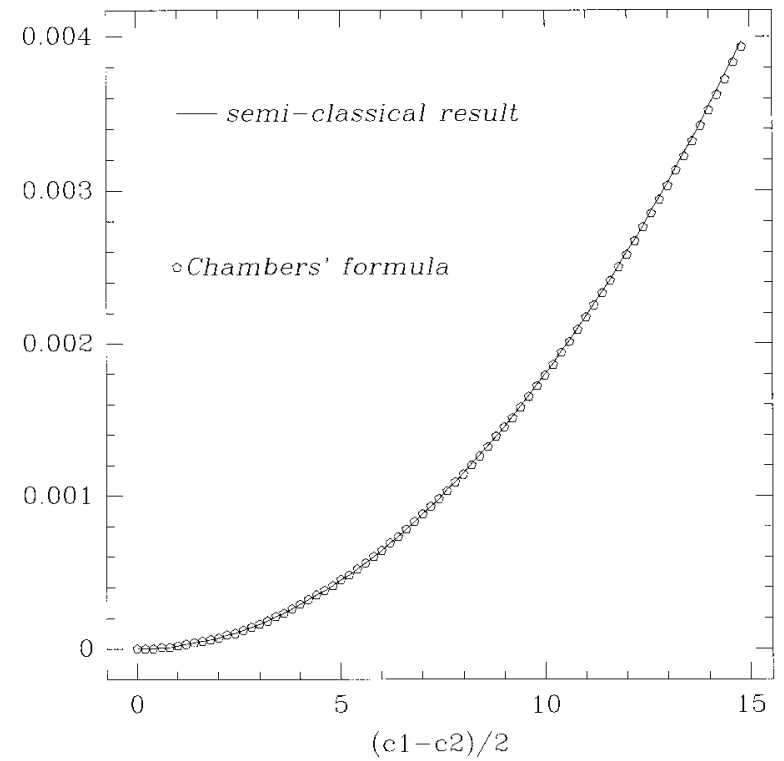

FIG. 9. Comparison between the semiclassical breakdown field $B_{0, i} / 4 \pi=\operatorname{Im}\left(f_{\beta}\right) / 4 B_{c}$ and Chambers' formula plotted as a function of $\left(c_{1}-c_{2}\right) / 2(\mathrm{meV})$ for the $\kappa$ phase.

the Brillouin zone, $\left(K_{x}, K_{y}\right)=(0,0)$. For energies above $\epsilon$ $\sim-100 \mathrm{meV}$, we begin to fill the other band, corresponding to the open surface.

We have made calculations of the de Haas-van Alphen spectrum by diagonalization of a finite lattice with a varying transverse magnetic flux. The presence of the flux breaks translational invariance, and the energy eigenvalues are found by a complete diagonalization of the lattice Hamiltonian (in practice, a $60 \times 60$ lattice) for each value of flux. The total energy is differentiated numerically with respect to flux to give the orbital magnetization. The method of calculation is outlined in Ref. 23. It is equivalent to what is sometimes referred to as "full quantum-mechanical calculation"-full in the sense that no semi-classical approximation is made for the magnetic flux. The three frequencies that appear in the de Haas-van Alphen spectrum (see Figs. 6 and 7) correspond to the area of the small closed orbit $\alpha$, and a greater orbit $\beta$, of unit area, obtained by a breakdown effect between the open and closed orbits. The third $(\beta-\alpha)$ has a total area equal to the difference between the areas of $\beta$ and $\alpha$ (the representation of the paths $\gamma_{\alpha}$ and $\gamma_{\beta}$ is indicated in Fig. 5). Such an orbit is classically problematic, as we shall discuss below.

Let us first construct the Riemann surface $M(P)$, the polynomial $P$ being deduced from the dispersion relation above. We therefore find two solutions $Z_{2}[\sigma]\left(Z_{1}\right)$ by solving for $\cos \left(K_{y}\right)$ in terms of $\cos \left(K_{x}\right)$. So, $M(P)$ consists of two

TABLE III. Fundamental de Haas-van Alphen frequencies for the two-band model presenting the structure of the organic compound $\kappa$-(BEDT-TTF $)_{2} \mathrm{Cu}(\mathrm{NCS})_{2}$, for two representative values of the parameter $\left(c_{1}-c_{2}\right)$.

\begin{tabular}{cccc}
\hline \hline$c_{1}-c_{2}$ & $\epsilon_{F}$ & $f_{\alpha} / B_{c}$ & $f_{\beta} / B_{c}$ \\
\hline 30 & -63.52 & 0.142 & $1+1.63 \times 10^{-2} i$ \\
2.5 & -60.05 & 0.157 & $1+4.47 \times 10^{-4} i$ \\
\hline \hline
\end{tabular}




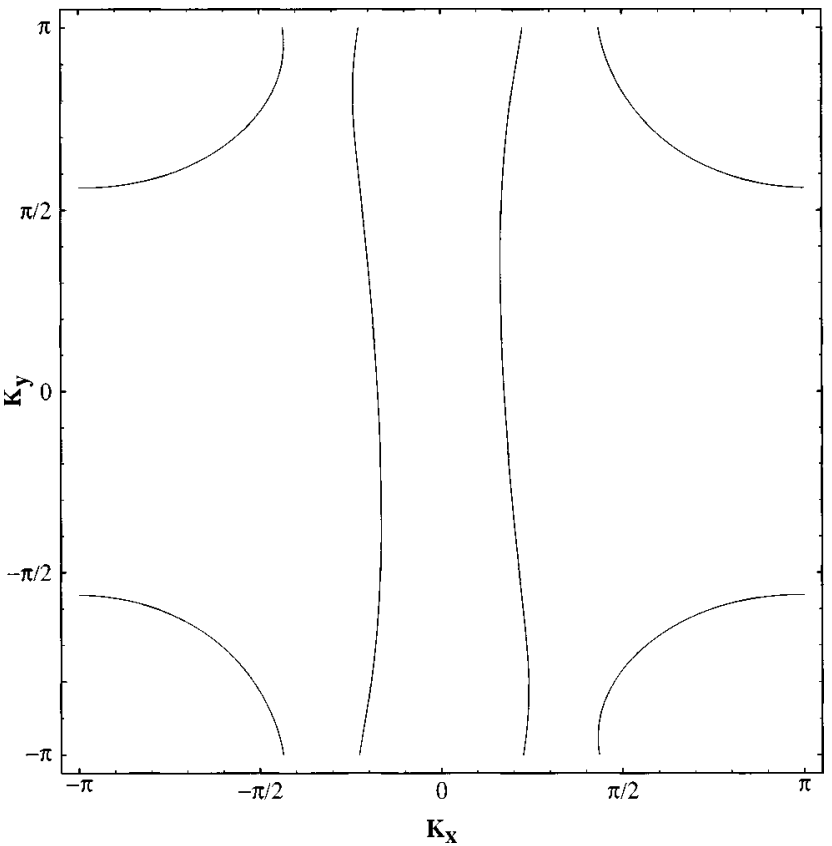

FIG. 10. Fermi surface of $\alpha$-(BEDT-TTF) ${ }_{2} \mathrm{MHgXCN}_{4}$ with the parameters calculated by Mori et al.

sheets (see Fig. 8) coming from the fact that to any fixed $K_{x}$ in the Brillouin zone there correspond two solutions $K_{y}$. There are eight singular points, with the same symmetry as the previous examples, $K_{x}$ and $K_{y}$ being expressed in terms of cosine functions. Each singular point has a ramification index equal to two, as $Z_{2}$ contains only square roots. There are also two polar singularities at $Z_{1}=-1,0$. The singular solutions, expressed as $Z_{1}\left[ \pm 1, \eta, \eta^{\prime}\right]$, are solutions of $X_{1}$ $=f\left[\eta, \eta^{\prime}\right]$, where

$$
\begin{aligned}
f\left[\eta, \eta^{\prime}\right]= & \frac{1}{2 b^{2}}\left(2 \epsilon_{F} b+\left(c_{1}+c_{2} \eta\right)^{2}+\eta^{\prime}\left(c_{1}+c_{2} \eta\right)\right. \\
& \left.\times \times \sqrt{4 \epsilon_{F} b+\left(c_{1}+c_{2} \eta\right)^{2}+8 b^{2}}\right),
\end{aligned}
$$

and the solutions \pm 1 are, as usual, ordered in decreasing value of real or imaginary parts. Their values are shown in Table II.

The last four points are given by the symmetry $Z_{1}\left[1, \sigma, \sigma^{\prime}\right]=1 / Z_{1}\left[-1, \sigma, \sigma^{\prime}\right]$. Topologically, $M(P)$ is a surface of genus 4 . The half path for the $\beta$ orbit from $P_{\alpha}$ to $P_{\beta}$ has been drawn in Fig. 8. This orbit includes four breakdown points, which occur for an appropriate representative path along the unit circle out of the arcs belonging to the real bands, between, for example, $Z_{1}[1,-1,-1]$ and $Z_{1}[1,1$, $-1]$. The numerical calculation has been performed at high field ( $B_{c} / B$ ranging from 5 to 55 ) with the choice of the parameter $\left(c_{1}-c_{2}\right)=30$ in order to resolve the three orbits in the spectrum. For small values of this parameter, as in the physical case, the gap between the $\alpha$ and $\beta$ orbits will be too weak for $\alpha$ to be observed at high field. By analytical computation we found the frequencies listed in Table III, which are in good agreement with the numerical spectrum. We also compare the Chambers' formula on one of the $\beta$-orbit breakdown points with the semiclassical calculus for varying $\left(c_{1}\right.$ $-c_{2}$ ) (see Fig. 9), which confirms that the agreement is almost perfect at small gaps. The case $c_{1}=c_{2}$ is special in the sense that we have curves crossing at the singular points $Z_{1}[\sigma,-1,1]=Z_{1}[\sigma,-1,-1]$. These points are removed from the Riemann surface as in the example of the previous section, and the genus is reduced to $2 . M(P)$ remains connected, since the polynomial cannot be factorized into two parts.

It is tempting to explain the existence of the frequency $\beta-\alpha$ in the topological formalism by the existence of a class of paths $\gamma_{\beta-\alpha}$, with the correct area $f_{\beta-\alpha}$, which could be drawn on the Riemann surface, Fig. 8. The imaginary part of such path is the same as $\beta$. The classical problem of this choice is that the hole apparently travels temporarily in the counterclockwise direction when it follows the small holepocket contours. This is rather troubling as it violates our classical intuition. In fact, what is required in a full semiclassical analysis are selection rules ${ }^{24}$ based on the conservation of current at each region of breakdown. This analysis is necessary to construct the correct frequency spectrum. The frequency $(\beta-\alpha)$ is absent from the fourier component of the frequency spectrum, but when we calculate the magnetization as a function of inverse field ${ }^{25}$ it appears as a result of imposing the constraint of constant total electron density. Thus the path drawn $\gamma_{\beta-\alpha}$ should not be taken literally and could even be omitted from the analysis as its imaginary part is the same as that of the path $\gamma_{\beta}$. If we estimated the breakdown field from the "unphysical" $\gamma_{\beta-\alpha}$ or the "physical", $\gamma_{\beta}$ we estimate the same breakdown field for the oscillation $(\beta-\alpha)$ so this point might seem academic. However if we attributed the occurrence of this frequency to the existence of the $\gamma_{\beta-\alpha}$ path we would then erroneously predict its appearance in other correlation functions, as a function of time at fixed field, for example. Thus we argue that the topological analysis we have given must be supplemented by selection rules, but contrary to the case of interference between different closed orbits, one still finds certain frequencies, $(\beta-\alpha)$ in particular, that naively might be excluded by arguing that the classical motion is in the "wrong" direction. Details of the calculation of the interference frequencies will be given elsewhere. ${ }^{25}$ Experimentally, the $\beta-\alpha$ oscillation is clearly seen in the magnetoresistance, ${ }^{26}$ with amplitudes higher than one expects from elementary arguments. Note, however, that here we are not calculating transport, and the question of interference is rather different for transport. ${ }^{6}$ In the case of de Haas-van Alphen oscillations, the question of the magnetization amplitude remains: a recent study ${ }^{27}$ found oscillations, but of weak amplitude, only a little larger than can be explained by demagnetization effects.

\section{BREAKDOWN FIELD COMPUTATION FOR THE TWO-DIMENSIONAL ORGANIC COMPOUND $\alpha-(\text { BEDT-TTF })_{2} \mathrm{MHgXCN}_{4}$}

Calculations for this family of compounds starting from molecular orbitals lead to a four-band tight-binding model. Each unit cell is an arrangement of four atoms, $a_{1}, a_{2}, b$, and $c$. The structure of interactions can be found in Ref. 28, with the calculated overlap coefficients (in $\mathrm{meV}$ ): $c_{1}=$ $-1.9, \quad c_{2}=6.8, \quad c_{3}=-1.1, \quad c_{4}=-1.4, \quad p_{1}=-10, \quad p_{2}=$ $-9.7, p_{3}=13.3$, and $p_{4}=13.2$. The four-dimensional wavefunction $\Psi=\left(\Psi_{a_{1}}, \Psi_{a_{2}}, \Psi_{b}, \Psi_{c}\right)$ then satisfies the Bloch equation $\hat{H} \Psi=\epsilon \Psi$, where 


$$
\hat{H}=\left(\begin{array}{cccc}
0 & c_{3} e^{-i K_{y}+c_{4}} & p_{1} e^{-i K_{x}}+p_{4} & p_{1} e^{-i K_{y}}+p_{4} e^{-i\left(K_{x}+K_{y}\right)} \\
c_{3} e^{i K_{y}}+c_{4} & 0 & p_{2} e^{-i K_{x}+p_{3}} & p_{2}+p_{3} e^{-i K_{x}} \\
p_{1} e^{i K_{x}}+p_{4} & p_{2} e^{i K_{x}}+p_{3} & 0 & c_{1} e^{-i K_{y}+c_{2}} \\
p_{1} e^{i K_{y}}+p_{4} e^{i\left(K_{x}+K_{y}\right)} & p_{2}+p_{3} e^{i K_{x}} & c_{1} e^{i K_{y}}+c_{2} & 0
\end{array}\right)
$$

The eigensolutions in energy are four independent bands. With three electrons per spin in the Brillouin zone, two of these bands are entirely filled. The third electron for each spin is shared between the last two bands, as in the previous example. We find the Fermi energy at $-17.2 \mathrm{meV}$. The contours of the Fermi surface (see Fig. 10) are qualitatively the same as the ones that we studied as the first example: there are four quarter-pocket areas at each corner of the Brillouin zone, and two open (or "quasi-one-dimensional") curves that cross the zone along $K_{y}$. Obviously, we have the single symmetry $\left(K_{x}, K_{y}\right) \rightarrow\left(-K_{x},-K_{y}\right)$. At low field, the only contribution to the magnetization oscillations are due to the closed-pocket areas. The question remains whether it is possible to see any magnetic breakdown between the pocket and the open surface at sufficiently low fields. Indeed, the numerical diagonalization at high fields suggested the existence of such a breakdown effect (see Fig. 11), with a frequency equal to the whole area of the first Brillouin zone ( $\beta$ orbit), in addition to the small frequency $\alpha$ at $f_{\alpha} / B_{c}=0.196$. The existence of a $\beta-\alpha$ orbit, that could be justified as in the previous section, was pointed out numerically in Ref. 23 , and in a similar study by Machida et al. ${ }^{29}$ Substituting in the eigenvalue equation $e^{i K_{j} \rightarrow Z_{j}}$ and $e^{-i K_{j}} \rightarrow 1 / Z_{j}$, we find a polynomial of degree 7 in $\left(Z_{1}, Z_{2}\right)$, the highest term being of the form $Z_{1}^{4} Z_{2}^{3}$. The highest degrees in $Z_{1}$ and $Z_{2}$ are 4 . As the coefficients of the polynomial are real, and $\hat{H}$ Hermitian, the following result holds: if $\left(Z_{1}, Z_{2}\right)$ is a solution, $\left(1 / Z_{1}, 1 / Z_{2}\right),\left(\bar{Z}_{1}, \bar{Z}_{2}\right)$, and $\left(1 / \bar{Z}_{1}, 1 / \bar{Z}_{2}\right)$ are also solutions. Now we want to compute the most singular points, in order to find a path to explain the existence of the $\beta$ orbit. As the polynomial cannot be solved explicitly, we studied numeri-

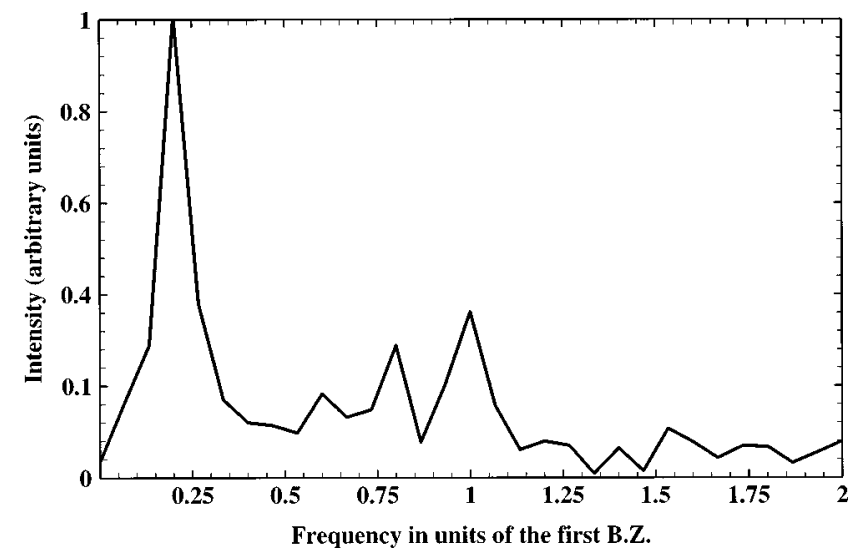

FIG. 11. Fourier spectrum of the magnetization of $\alpha$-(BEDT-TTF $)_{2} \mathrm{MHg}(\mathrm{XCN})_{4}$ as a function of $B_{c} / B$, as calculated on the lattice. The primary breakdown frequency $\beta$ is at $100 \%$ of the area of the first Brillouin zone. Also visible are the peak corresponding to the hole pocket at frequency $\alpha=19.6 \%$ and the interference frequency $\beta-\alpha$. cally the solutions of $P=0$ and $\partial_{Z_{1}} P=0$. The choice of differentiating $P$ with respect to $Z_{1}$ instead of $Z_{2}$ is purely for convenience. Indeed, it allows fewer band edges on the unit circle of the Riemann surface. The resolution of $\partial_{Z_{1}} P=0$ provides us with exact expressions of $Z_{1}=Z_{1}\left(Z_{2}\right)$ as three solutions of a polynomial of third degree. Then, replacing $Z_{1}$ in $P$, we have an algebraic equation in $Z_{2}$ that we can solve numerically. Inside the upper unit circle, we found seven values for $Z_{2}$, listed in Table IV. The others can be deduced by the symmetries $Z_{2} \rightarrow 1 / Z_{2}, Z_{2} \rightarrow \bar{Z}_{2}$. We do not give the corresponding $Z_{1}$ values. $P_{1}$ corresponds to the unique band edge of the closed areas. Obviously, there is no band edge for the open parts in the $K_{y}$ direction. $P_{2}$ defines the bridge between the closed areas and the open parts. The other points are too close to the origin (or, symmetrically, to the point at infinity) to play an important role in the breakdown effect. The path we consider for the breakdown piece (see Fig. 12) is the following: we begin at $\left(Z_{1}, Z_{2}\right)=\left(-1, P_{1}\right)$, and solve for $Z_{1}$ at each point of the parametric continuous path

$$
\begin{gathered}
\gamma(t)=\left(Z_{1}(t), Z_{2}(t)\right), \quad 0 \leqslant t \leqslant 2, \\
Z_{2}(t)=\left(P_{2}-P_{1}\right) t+P_{1}, \quad 0 \leqslant t \leqslant 1, \\
Z_{1}(1) \simeq 0.519-0.757 i, \\
Z_{2}(t)=\left(-1-P_{2}\right)(t-1)+P_{2}, \quad 1 \leqslant t \leqslant 2, \\
Z_{1}(2) \simeq 0.758-0.652 i .
\end{gathered}
$$

At $t=1$ we have to make a change of $Z_{2}$ identification as we jump from one of the four sheets to another. This path is a small part of the complete $\beta$-orbit action. In fact, we only need to calculate the numerical value of the imaginary part of this action. The real part can in fact be seen to be exactly 1 from a symmetry argument: the contribution from the product of real parts of the $\mathbf{k}$ vectors gives 1 from stoichiometry, and the contributions from the imaginary parts can-

TABLE IV. Most singular points on the Riemann surface corresponding to the energy dispersion for the two-dimensional tightbinding model associated with the organic compound $\alpha$-(BEDT-TTF $)_{2} \mathrm{MHgXCN}_{4}$.

\begin{tabular}{lll}
\hline \hline & $\operatorname{Re}\left(Z_{2}\right)$ & $\operatorname{Im}\left(Z_{2}\right)$ \\
\hline$P_{1}$ & -0.192 & 0.981 \\
$P_{2}$ & -0.477 & 0.161 \\
$P_{3}$ & -0.0236 & 0.00629 \\
$P_{4}$ & -0.138 & 0 \\
$P_{5}$ & 0.00883 & 0 \\
$P_{6}$ & 0.00869 & 0 \\
$P_{7}$ & 0.000812 & 0 \\
\hline \hline
\end{tabular}




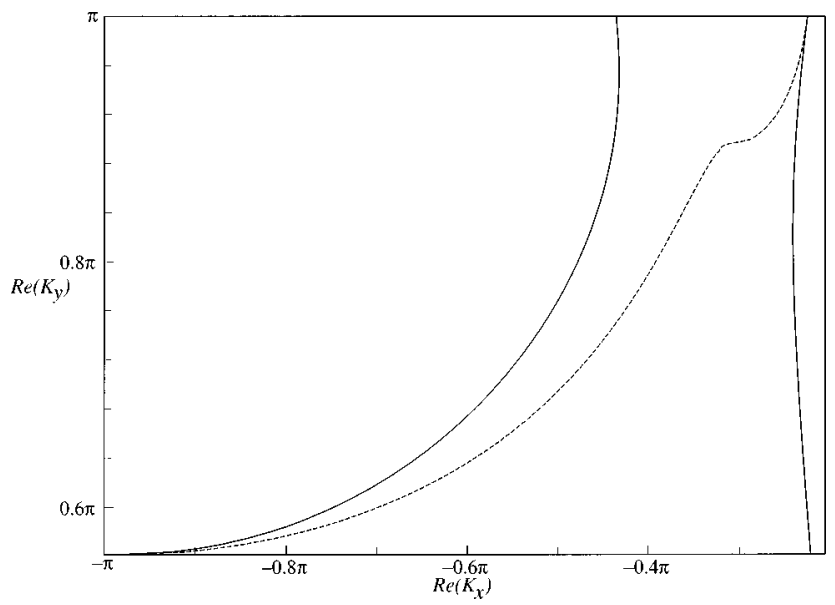

FIG. 12. A part of the projection of one possible breakdown path (dashed lines) onto real $\mathbf{k}$ space.

cel by symmetry. Four magnetic breakdowns occur along the orbit. We finally have $f_{\beta} / B_{c} \simeq 1+0.0333 i$, giving magnetization oscillations corresponding to the area of the first Brillouin zone damped with a characteristic field of $0.209 B_{c}$. This can be compared with the result applying Chambers' formula: $0.202 B_{c}$. Thus, from the value of $B_{c}$ we predict $880 \mathrm{~T}$ for the breakdown field, based on the band structure. This seems to exclude a single-particle tunneling effect: if breakdown has been seen, as is possibly the case, ${ }^{30}$ it seems that either the band structure is not accurately giving the gap or there is a collective effect. We note that recent recalculations of the band structure, all based on the extended Hückel approach, by Ducasse ${ }^{31}$ and Canadell ${ }^{32}$ give slightly different parameters, but they do not vary enough to explain any observed breakdown. For example, using Ducasse's parameters, Chambers' formula gives a slightly smaller value, 0.0280, in units of the first-Brillouin-Zone area, compared to Mori's 0.0333 and Canadell's (at $104 \mathrm{~K}$ ) 0.0304. We conclude that breakdown cannot be a simple tunneling in the original Fermi surface, and this is in agreement with the interpretations of McKenzie et al. ${ }^{15}$ that the breakdown is from some, as yet undetermined, reconstructed state.

\section{CONCLUSIONS}

We have shown how we can make systematic calculations of magnetic breakdown in situations where there are several sheets to the band structure. Our algebraic approach rewrites the energy dispersion relation as a polynomial form involving complex variables constructed from the components of the momentum. This is then used to incorporate an applied magnetic field via the Peierls' substitution. The restriction to a finite-order polynomial is very mild in physical terms, and can be tested by including more sheets. In general, this seems unlikely to alter the results greatly, at least for breakdown fields that are small enough to be measurable, given that the extra sheets do not contribute to the Fermi surface. A possible exception to this may be, for example, in the case of aperiodic solids, where the Fermi surface should really involve an infinite number of sheets. But even this case may be tractable by taking the appropriate limit of a finite-order calculation. In practice, the approach seems most useful in cases, such as the molecular conductors, where extended Hückel calculations are good starting points to understand the electronic structure, and lead to rather low-order polynomials. As we have seen, the difficulty of the calculation will increase with the order of the polynomial, mainly because the topology becomes more involved. The need to solve the algebraic equation numerically is not an obstacle. It appears that a semiclassical analysis will suffice to explain observed features in numerical studies of "full" quantum-mechanical calculations. While there is not a large discrepancy between the breakdown fields predicted here and those found from the semiempirical approach of Chambers, this is an advance at least in the reliability of the results. We have discussed a case, the limit of vanishing hybridization in a two-band model, where naive application of Chambers' formula can give a wrong result.

The topological analysis described allows us to systematically calculate the fundamental frequencies and damping of all possible magnetic oscillations. Determining which ones can be actually observed requires additional selection rules related to current conservation. The semi-classical approach should allow more detailed calculation of what may be called " 'interference effects,' namely, frequencies that correspond to sums and differences of areas. The analysis of this aspect is not yet complete. For instance, we have not considered the calculation of effective masses. We have, however, shown how to calculate the dominant part of the tunneling probabilities, which are the starting point of such an analysis. Precise resolution of questions involving sums and differences of the primary frequencies in the magnetic oscillations may be useful, ultimately, in order to show, for example, the limitations of the single-electron picture.

\section{ACKNOWLEDGMENTS}

M.G. acknowledges support from the Brazilian-French agreement CAPES/COFECUB 196/96.
${ }^{1}$ D. Shoenberg, Magnetic Oscillations in Metals (Cambridge University Press, Cambridge, 1984).

${ }^{2}$ M. H. Cohen and L. M. Falicov, Phys. Rev. Lett. 7, 231 (1961).

${ }^{3}$ E. I. Blount, Phys. Rev. 126, 1636 (1962).

${ }^{4}$ A. B. Pippard, Proc. Phys. Soc. London A270, 1 (1962).

${ }^{5}$ R. G. Chambers, Proc. Phys. Soc. London 88, 701 (1966).

${ }^{6}$ R. W. Stark and Friedberg, Phys. Rev. Lett. 26, 556 (1971).

${ }^{7}$ M. Gusmão and T. Ziman, Phys. Rev. B 54, 16663 (1996).

${ }^{8}$ H. A. Kramers, Z. Phys. 39, 928 (1926).

${ }^{9} \mathrm{~V}$. Arnold, Mathematical Methods of Classical Mechanics, 2nd ed. (Springer Verlag, New York, 1989), p. 196.

${ }^{10}$ H. A. Fertig and B. I. Halperin, Phys. Rev. B 36, 7969 (1987).

${ }^{11}$ J. M. Luttinger, Phys. Rev. 84, 814 (1951).

${ }^{12}$ R. E. Peierls, Quantum Theory of Solids (Oxford University, Oxford, 1956).

${ }^{13}$ T. Sasaki, H. Sato, and N. Toyota, Physica C 185-189, 2687 (1991).

${ }^{14}$ J. Wosnitza et al., Phys. Rev. B 45, 3018 (1992).

${ }^{15}$ R. H. McKenzie, G. J. Athas, J. S. Brooks, R. G. Clark, A. S. Dzurak, R. Newbury, R. P. Starrett, A. Skougarevsky, M. Toku- 
moto, N. Kinoshita, T. Kinoshita, and Y. Tanaka, Phys. Rev. B 54, R8289 (1996).

${ }^{16}$ I. M. Lifshitz and A. M. Kosevich, Zh. Eksp. Teor. Fiz. 20, 739 (1955) [Sov. Phys. JETP 2, 636 (1956)].

${ }^{17}$ L. M. Falicov and H. Stachowiak, Phys. Rev. 147, 505 (1966).

${ }^{18}$ L. M. Roth, Phys. Rev. 145, 434 (1966).

${ }^{19}$ B. A. Dubrovin, A. Fomenko, and S. Novikov, Modern Geometry-Methods and Applications: Part 2. The Geometry and Topology of Manifolds (Springer Verlag, Berlin, 1985).

${ }^{20} \mathrm{~B}$. Chabat, Introduction à L'analyse Complexe (Mir, Moscow, 1990), Vol. 1; Rick Miranda Algebraic Curves and Riemann Surfaces, Graduate Studies in Mathematics, Vol. 5 (American Mathematical Society, 1995).

${ }^{21}$ J. Caufield et al., J. Phys.: Condens. Matter 6, 2911 (1994).

${ }^{22}$ C. E. Campos, P. S. Sandhu, J. Brooks, and T. Ziman, Phys. Rev. B 53, 12725 (1996).

${ }^{23}$ R. Fleckinger, M. Gusmão, Y. Tan, and T. Ziman, in Physical Phenomena at High Magnetic Fields-II, edited by Z. Fisk, L. Gorkov, D. Meltzer, and R. Schrieffer (World Scientific, Singapore, 1996), p. 776.

${ }^{24}$ A. A. Slutskin, Zh. Eksp. Teor. Fiz. 53, 767 (1967) [Sov. Phys. JETP 26, 474 (1968)].
${ }^{25}$ J. Y. Fortin and T. Ziman (unpublished).

${ }^{26}$ C. C. Agosta, C. H. Mielke, S. A. Ivanov, D. Howe, J. D. Goettee, M. Tokumoto, Y. Tanaka, and N. Kinoshita, in Physical Phenomena at High Magnetic Fields-II, edited by Z. Fisk, L. Gorkov, D. Meltzer, and R. Schrieffer (World Scientific, Singapore, 1996), p. 297.

${ }^{27}$ F. A. Meyer, E. Steep, W. Biberacher, P. Christ, A. Lerf, A. G. M. Jansen, W. Joss, P. Wyder, and K. Andres, Europhys. Lett. 32, 681 (1995).

${ }^{28}$ H. Mori, S. Tanaka, M. Oshima, G. Saito, T. Mori, Y. Murayama, and H. Inokuchi, Bull. Chem. Soc. Jpn. 57, 627 (1984).

${ }^{29}$ K. Machida, K. Kishigi, and Y. Hori, Phys. Rev. B 51, 8946 (1995).

${ }^{30}$ S. Uji, H. Aoki, J. S. Brooks, A. S. Perel, G. J. Athas, S. J. Klepper, C. C. Agosta, D. A. Howe, M. Tokumoto, N. Kinoshita, Y. Tanaka, and H. Anzai, Solid State Commun. 88, 683 (1993).

${ }^{31}$ L. Ducasse and A. Fritsch, Solid State Commun. 91, 201 (1994).

${ }^{32}$ R. Rousseau, M.-L. Doublet, E. Canadell, R. P. Shibaeva, S. S. Khasanov, L. P. Rozenberg, N. D. Kushch, and E. B. Yagubskii, J. Phys. I 6, 1527 (1996). 